\title{
Expeditious synthesis of helicenes using an improved protocol of photocyclodehydrogenation of stilbenes
}

\author{
Harish R. Talele, Anju R. Chaudhary, Parthiv R. Patel, \\ and Ashutosh V. Bedekar* \\ Department of Chemistry, Faculty of Science \\ The Maharaja Sayajirao University of Baroda, Vadodara 390 002, India \\ E-mail: avbedekar@yahoo.co.in
}

\begin{abstract}
An improved procedure has been developed for photodehydrocyclization of stilbenes for the synthesis of phenanthrenes and helicenes. This procedure involves the use of THF as a scavenger of hydriodic acid produced during iodine mediated photodehydrocyclization. The use of THF is advantageous due to its higher boiling point, lower cost and easy availability as compared to propylene oxide. The method is applied to synthesize a number of phenanthrenes and helicenes.
\end{abstract}

Keywords: Helicenes, HI scavenger tetrahydrofuran, Mizoroki-Heck-reaction, Phenanthrenes, Stilbenes, Wittig-olefination

\section{Introduction}

Helicenes ${ }^{1}$ constitute a fascinating class of chiral helical molecules comprising ortho-fused aromatic rings having many intriguing features such as extended aromaticity, chirality, a capability to organize into columnar solid state architecture and an ability to behave as organic conductors. Potential applications of helicenes can be found in the fields of non-linear optics ${ }^{2}$ and circularly polarized luminescence. ${ }^{3}$ The unique structure of functionalized helicenes make them very stable towards acids, bases as well as being stable at high temperature. ${ }^{4}$ These type of molecules are considered potentially useful new materials such as discotic liquid crystals ${ }^{5}$ or conjugated polymers. ${ }^{6}$ Study of helical compounds is an active field of research in supramolecular chemistry due to their self-assembly and physiochemical properties. ${ }^{7}$ Also their rigid helical framework, high optical stability and unique chiral array can provide functionalized helicenes such as alcohols, ${ }^{8}$ nitriles, ${ }^{9}$ amines ${ }^{10}$ and phosphines ${ }^{11}$ for use as chiral catalysts, ${ }^{12}$ ligands ${ }^{13}$ and auxiliaries in asymmetric synthesis. Moreover helicenes possessing inherent chirality have attracted attention owing to their extraordinary electronic and optical properties. ${ }^{14}$ 
A common method for the synthesis of phenanthrene and its derivatives involves the construction of a central ring by photodehydrocyclization of stilbene. This method involves exposure of cis/trans stilbene $\mathbf{1}$ to UV light, which causes its isomerization to the cis form. This form undergoes electrocyclic ring closure to produce dihydrophenanthrene $\mathbf{2}$, which on dehydrogenation generates a phenanthrene $\mathbf{3}$ (Scheme 1).

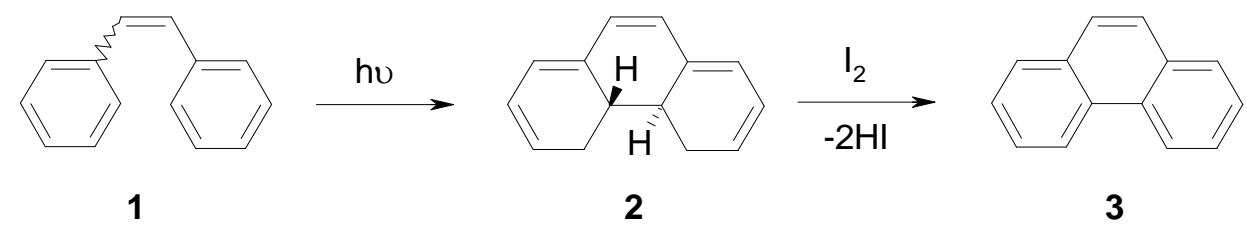

Scheme 1. Transformation of cis-stilbene 1 into phenanthrene 3.

This classical synthesis of ortho-fused benzene rings has been optimized and widely applied for the syntheses of a number of helical moieties. ${ }^{15}$ The required stilbene precursors are obtained either by the Wittig reaction or by the Mizoroki-Heck reaction. In recent years, preparation of heterohelicenes has been extensively studied in order to exploit the unique properties of these compounds. ${ }^{16}$

\section{Results and Discussion}

As a part of our ongoing research project, we require a simple an expeditious synthesis of functionalized benzo[c]phenanthrene. In the preliminary communication ${ }^{17}$ we have reported an improved method for photocyclodehydrogenation of stilbenes to construct phenanthrenes, helicenes etc. In the classical iodine mediated photocyclodehydrogenation, propylene oxide is used as a scavenger to neutralize the co-product hydrogen iodide. This reagent is slightly expensive and has a low boiling point. In a typical photo reaction even under careful cooling, the reaction mixture warms up which causes the loss of propylene oxide. In our improved procedure we have replaced this scavenger by readily available and cheap tetrahydrofuran which has a higher boiling point and in this paper we present further applications of this modified procedure for the synthesis of a number of helical compounds.

The progress of the neutralization of hydrogen iodide with tetrahydrofuran and propylene oxide is studied by $\mathrm{pH}$ measurement of its solution. In this study tetrahydrofuran and propylene oxide neutralizes the HI due to the opening of both cyclic ethers. As a result of the neutralization of HI there is a gradual rise in the $\mathrm{pH}$. We found that the propylene oxide reacts faster than the tetrahydrofuran, Figure 1. This is expected since the propylene oxide has a higher strain compared to tetrahydrofuran, nevertheless this study indicates clearly that even though less efficiently, the later can be used as a scavenger of HI. The ability of tetrahydrofuran to react with $\mathrm{HI}$ is also reported in the literature. ${ }^{18}$ 


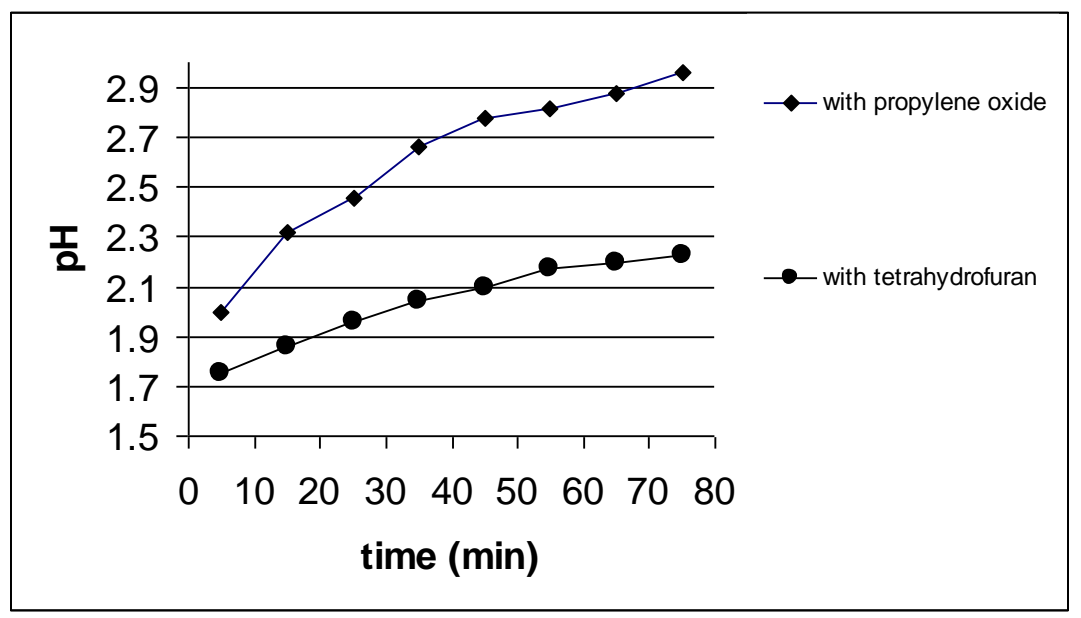

Figure 1. Comparison of propylene oxide and tetrahydrofuran as scavenger of HI.

In this paper we report several photodehydrocyclization reactions using tetrahydrofuran as scavenger in stead of propylene oxide and hope to establish the generality of this method. With this aim several suitable stilbenes were prepared by various methods and subjected to photodehydocyclization using the present conditions by which a number of mono- and disubstituted phenanthrenes were prepared in excellent yields ${ }^{17}$ (Scheme 2).

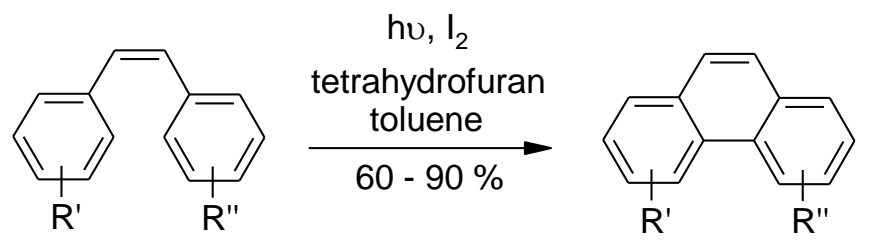

$\mathrm{R}^{\prime}$ and $\mathrm{R}^{\prime \prime}=\mathrm{H}, \mathrm{Me}, \mathrm{Cl}, \mathrm{Br}, \mathrm{OMe}$

Scheme 2. Improved synthesis of substituted phenanthrenes.

The photodehydrocyclization of nitrostilbenes is known to offer some difficulty when a catalytic quantity of iodine is used. ${ }^{19}$ In order to test the present method on the nitro containing substrates, stilbenes 1a and $\mathbf{1 b}$ were synthesized and subjected to photolysis with $\mathrm{I}_{2}$-THF. The corresponding nitro phenanthrenes $\mathbf{2 a}$ and $\mathbf{2 b}$ were isolated in reasonable yields (Scheme 3). Furthermore, the nitro derivative of styrylnaphthalene (2-[2-(4-nitrophenyl)ethenyl]naphthalene) ${ }^{20} \mathbf{3}$ was also successfully cyclized to 2-nitrobenzo[c]phenanthrene $\mathbf{4}$ indicating that the present method also tolerates nitro groups. 
<smiles>[R]c1ccc(/C=C/c2ccc([N+](=O)[O-])cc2)cc1</smiles>

1a; $\mathrm{R}=\mathrm{H}$

1b; $\mathrm{R}=\mathrm{Br}$<smiles>O=[N+]([O-])c1ccc(/C=C/c2ccc3ccccc3c2)cc1</smiles>

3 hu, I2, THF, Toluene

125 W HPMVL<smiles>[R]c1ccc2ccc3ccc([N+](=O)[O-])cc3c2c1</smiles>

2a; $\mathrm{R}=\mathrm{H} \quad(31 \%)$

2b; $R=B r(68 \%)$

hv, l2, THF, Toluene

125 W HPMVL, $29 \mathrm{~h}$<smiles>O=[N+]([O-])c1ccc2ccc3ccc4ccccc4c3c2c1</smiles>

$4(83 \%)$

Scheme 3. Preparation of nitro-substituted angularly fused arene.

The present method was screened for cyclization of 1,2-bis(2-naphthyl)ethylene 5 with the aim to prepare [5]helicene. However, the initially formed [5]helicene 6 was not isolated but underwent further $4 \pi+2 \pi$ cyclization to form benzo[ghi]perylene 7 in good yield (Scheme 4$){ }^{21}$<smiles>C1=Cc2ccccc2/C1=C\c1ccc2ccccc2c1</smiles>

5

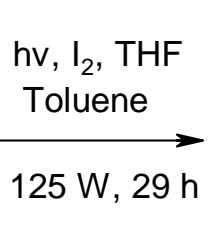

6<smiles></smiles>

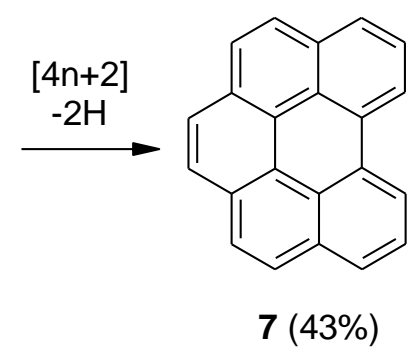

Scheme 4. Attempted synthesis of [5]helicene.

Since the photochemical ring closure had proven to be one of the simplest ways to obtain fused aromatics, we decided to study the reaction of different derivatives of ethylene, namely the compounds carrying a fused two- or three-ring aryl on one terminus and a monocyclic aryl on the other terminus of the double bond. The synthesis of these derivatives easily gave the desired helicenes and eventually opened the facile route for the formation of [6]helicenes or larger helicenes.

Encouraged by the previous results, several [6]helicene derivatives have been synthesized as shown in scheme 5. Readily accessible 2-naphthaldehyde 8 was converted into 2-(4bromostyryl)naphthalene $\mathbf{1 0}$ by reaction with the Wittig salt (4-bromobenzyl)triphenylphosphonium 
bromide 9 under standard conditions. The photodehydrocyclization of $\mathbf{1 0}$ in toluene with iodineTHF gave 2-bromobenzo[c]phenanthrene 11 in high yield.

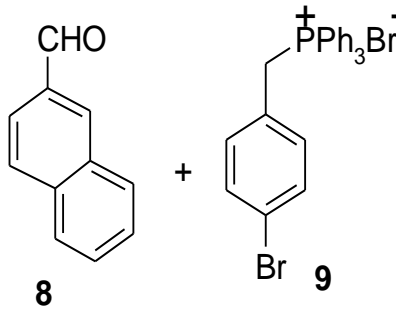

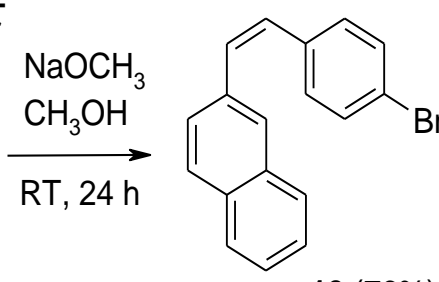

$10(76 \%)$

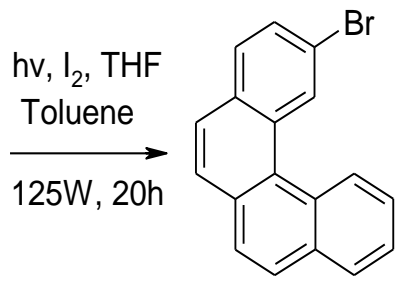

$11(96 \%)$

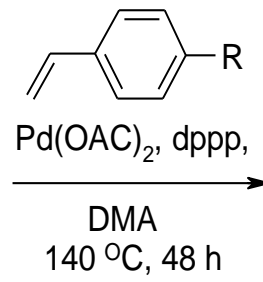

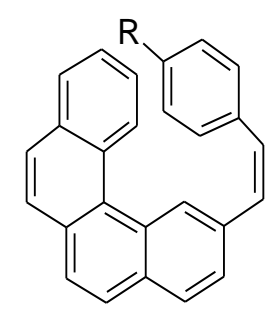

12; $\mathrm{R}=\mathrm{H} \quad(98 \%)$

13; $\mathrm{R}=\mathrm{CH}_{3}(95 \%)$
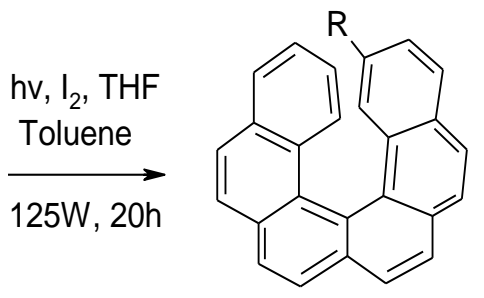

$14 ; \mathrm{R}=\mathrm{H} \quad(64 \%)$

$15 ; \mathrm{R}=\mathrm{CH}_{3}(78 \%)$

Scheme 5. Synthesis of [6]helicenes.

Reaction of 2-bromobenzo[c]phenanthrene 11 with styrene or 4-methylstyrene using the wellknown $\mathrm{Pd}(\mathrm{OAc})_{2}$-1,3-bis(diphenylphosphino)propane (Pd-dppp) catalyzed Heck reaction ${ }^{22}$ resulted into 2-styrylbenzo[c]phenanthrene 12 and 2-(4-methylstyryl)benzo[c]phenanthrene 13 respectively in excellent yield. Further photoirradiation of $\mathbf{1 2}$ or $\mathbf{1 3}$ under the present reaction conditions furnished [6]helicene 14 and 2-methyl[6]helicene 15 respectively in good yield.

Alternatively 14 can be also synthesized by double photodehydrocyclization of 2,7-bisstyrylnaphthalene 18. This new route is presented in scheme 6, where initially 2,7dihydroxynaphthalene $\mathbf{1 6}$ is converted into the corresponding 2,7-dibromonaphthalene 17, which was reacted with styrene under Heck conditions with Pd-dppp to afford 2,7-bis-styrylnaphthalene 18. This was then subjected to the photodehydrocyclization to afford [6] helicene $\mathbf{1 4}$ in a single step (Scheme 6).

The method is further studied for the syntheses of [7]helicene. Scheme 7 illustrates an attempt for its synthesis. The readily available 2-methylnaphthalene 19 was converted to its Wittig salt 20 via benzylic bromination with NBS, and its reaction with triphenylphosphine. The salt 20, on Wittig reaction with 4-tolualdehyde gave mostly the trans isomer of 2-(4-methylstyryl)naphthalene 21 in good yield, which upon photoirradiation resulted into 2-methylbenzo[c]phenanthrene 22. Similarly 22 was converted to the new Wittig salt 23, via its bromomethyl derivative. Standard Wittig reaction of $\mathbf{2 3}$ with 2-naphthaldehyde yielded 2-(2-vinylnaphthyl)benzo[c]phenanthrene $\mathbf{2 4}$, predominantly in the trans form. Photodehydrocyclization of $\mathbf{2 4}$ led to an unexpected result as the ring closure was directed to the less crowded site yielding the linear derivative, dinaphtho[1,2- 
$a: 2 ', 1 '-h$ ]anthracene 25 instead of the expected [7] helicene 26. The structure of compound 25 was established by comparison of spectroscopic data and its melting point. ${ }^{23}$<smiles>Oc1ccc2ccc(O)cc2c1</smiles>

16

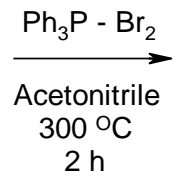<smiles>Brc1ccc2ccc(Br)cc2c1</smiles>

$17(53 \%)$

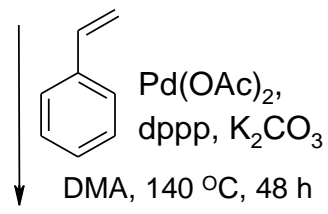<smiles></smiles>

$14(75 \%)$

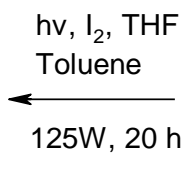<smiles>C(=C\c1ccc2ccc(/C=C\c3ccccc3)cc2c1)\c1ccccc1</smiles>

$18(65 \%)$

Scheme 6. Another approach to [6]helicene.<smiles>Cc1ccc2ccccc2c1</smiles>

19 a) hu, NBS, Bezoyl peroxide $\mathrm{CCl}_{4}, 8$

b) $\mathrm{PPh}_{3}$, Xylene $12 \mathrm{~h}$<smiles>[18O-]c1ccc2ccccc2c1</smiles>

$20(70 \%)$<smiles>Cc1ccc(/C=C\c2ccc3ccccc3c2)cc1</smiles>

21 (86\%)

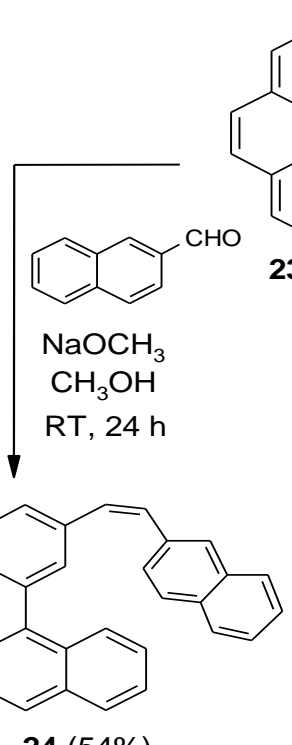

a) hu, NBS, bezoyl peroxide $\mathrm{CCl}_{4}, 8 \mathrm{~h}$

b) $\mathrm{PPh}_{3}$, Xylene $15 \mathrm{~h}$<smiles>Cc1ccc2ccc3ccc4ccccc4c3c2c1</smiles>
22 (96\%)

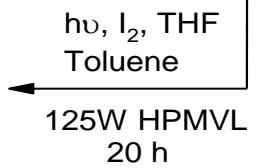
$23(70 \%)$

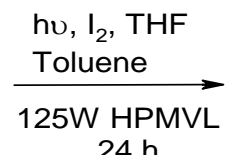

$24 \mathrm{~h}$<smiles>c1ccc2c(c1)ccc1cc3c(ccc4ccc5ccccc5c43)cc12</smiles>

$25(75 \%)$

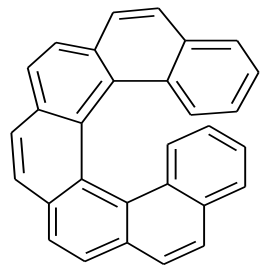

26 (not detected)

Scheme 7. Towards [7]helicene. 
The presence of the bulky naphthalene ring may prevent cyclization of $\mathbf{2 4}$ at the required angular location. In a modified route the double photocyclization/dehydrogenation was attempted starting from 30 where the bulky naphthyl group (as present in 24) had been replaced by the less bulky phenyl ring (Scheme 8). Accordingly, the required 3,6-(bis-styryl)phenanthrene 30 was synthesized by combination of Witting reaction, photodehydrocyclization and Heck reaction starting from 4-bromobenzaldehyde 27. Photodehydrocyclization of $\mathbf{3 0}$ under our improved conditions gave the desired [7] helicene $\mathbf{2 6}$ although in moderate yield.

In connection with our project on the synthesis of substituted [7]helicenes, a process for making 9,10-dialkoxy[7]helicenes was needed. With this aim, a commercial sample of phenanthrene 31 was converted to 9,10-phenanthrenequinone by oxidation, followed by conversion to 3,6-dibromo-9,10-phenanthrenequinone 32 by bromination. The required 3,6-dibromo-9,10dimethoxyphenanthrene 33 was prepared by reduction of 32 using $\mathrm{Na}_{2} \mathrm{~S}_{2} \mathrm{O}_{4}$ followed by a in situ Omethylation using dimethyl sulfate. The desired $\mathrm{Pd}(\mathrm{OAc})_{2}$ catalyzed double Heck reaction of $\mathbf{3 3}$ with styrene to get 3,6-(bis-styryl)-9,10-dimethoxyphenanthrene 34 proved to be difficult with dppp but succeeded with an 1-( $\alpha$-aminobenzyl)-2-naphthol ligand. ${ }^{24}$ Subsequent photodehydrocyclization of $\mathbf{3 4}$ gave 9,10-dimethoxy[7]helicene $\mathbf{3 5}$ in moderate yield (Scheme 9).

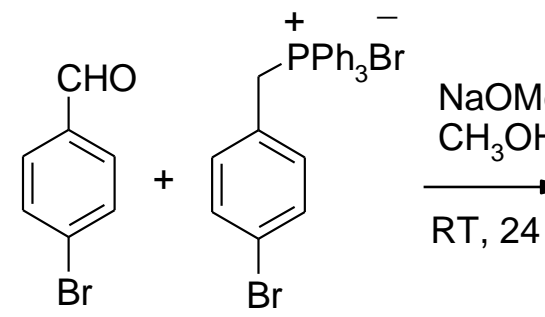

27<smiles>Brc1ccc(/C=C\c2ccc(Br)cc2)cc1</smiles>

$28(91 \%)$

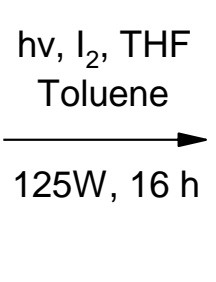<smiles>Brc1ccc2ccc3ccc(Br)cc3c2c1</smiles>

$29(97 \%)$

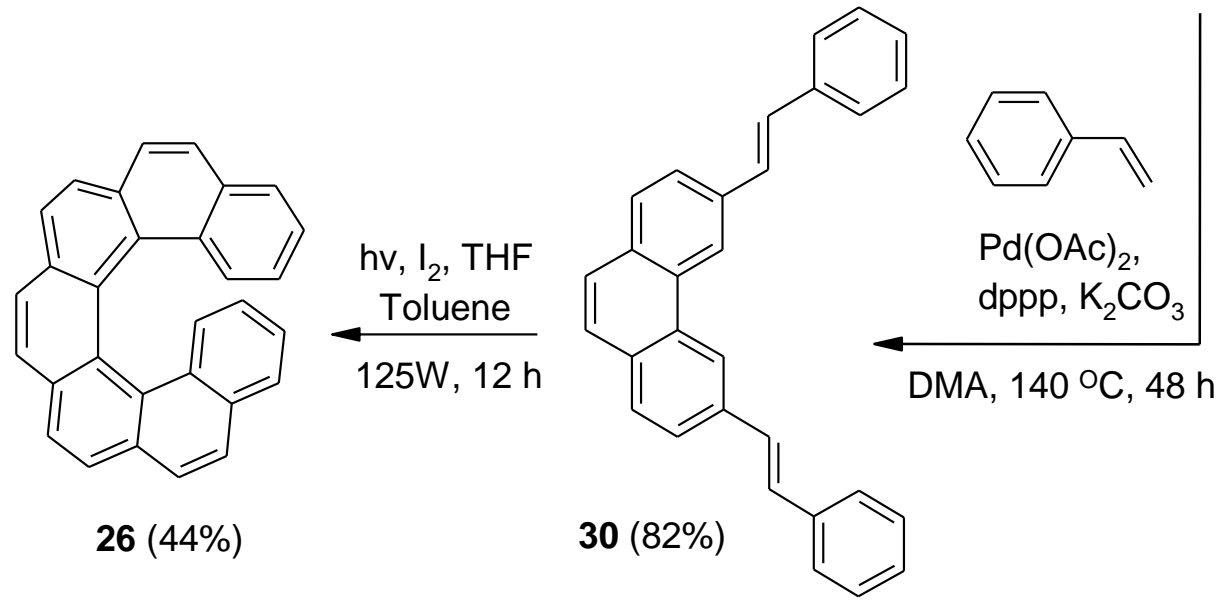

Scheme 8. Synthesis of [7]helicene. 


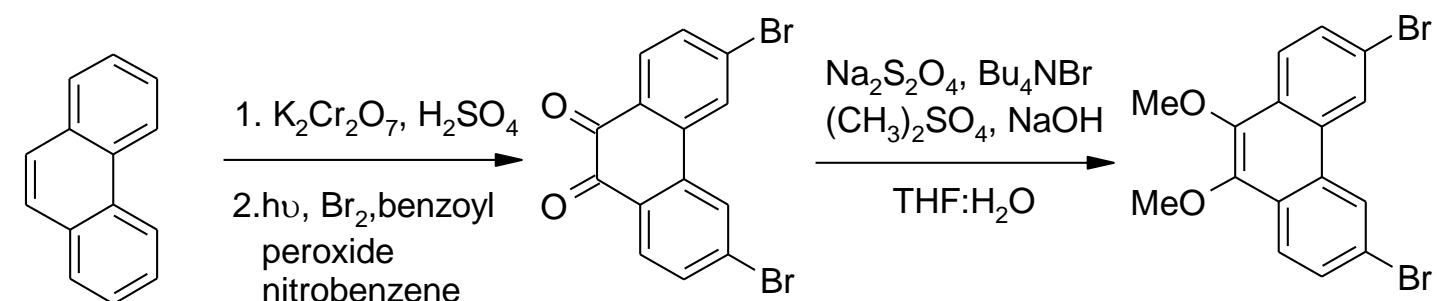

31 $70^{\circ} \mathrm{C}, 3 \mathrm{~h}$

$32(73 \%)$<smiles>COc1c(OC)c2ccc3ccc4ccc5ccccc5c4c3c2c2c1ccc1ccccc12</smiles>

Scheme 9. Synthesis of 9,10-dimethoxy[7]helicene.

Table 1. Comparison of photodehydrocyclization of styryl derivatives in presence of tetrahydrofuran and propylene oxide

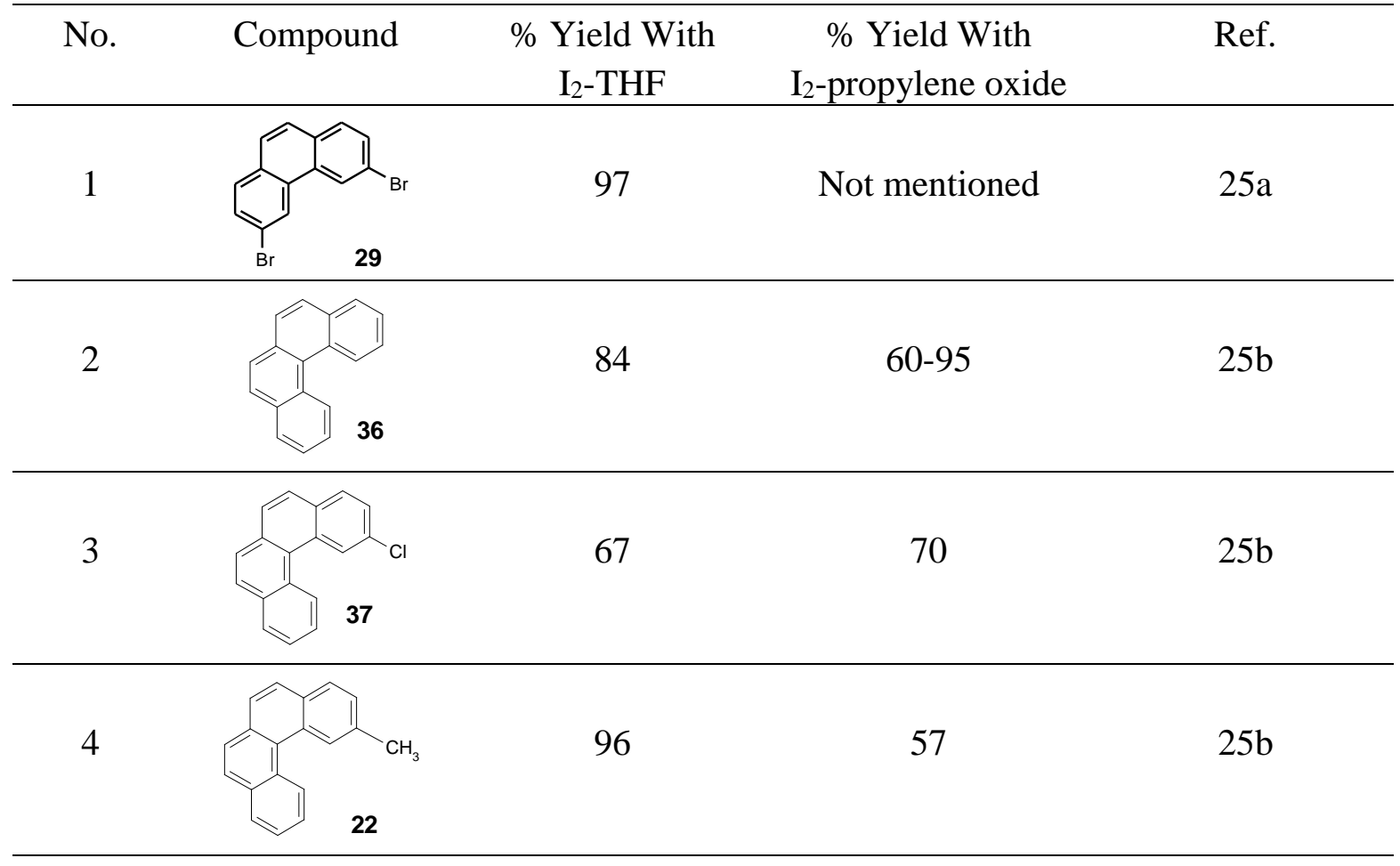


Table 1. (Continued)

(4)

\section{Experimental Section}

Reagents were purchased from Sigma-Aldrich Chemicals Limited, SD Fine, Sisco, Qualigens Limited etc. Tetrahydrofuran was refluxed and distilled on sodium benzophenone-ketyl. Toluene was distilled and stored $24 \mathrm{~h}$ over molecule sieves $4 \AA$ prior to use. Thin Layer Chromatography was performed on Merck $60 \mathrm{~F}_{254}$ Aluminium coated plates. The spots were visualized under UV light or with iodine vapour. Photo reactions were performed in immersion well photo reactor with water jacket for cooling with $125 \mathrm{~W}$ or $250 \mathrm{~W}$ high pressure mercury vapor lamp made by General Electric (CEMA Electric Lighting Products India Pvt. Ltd).

All the compounds were purified by column chromatography using SRL silica gel (60-120 mesh). ${ }^{1} \mathrm{H}$ NMR spectra were recorded on Bruker Avance 400 Spectrometer and were run in $\mathrm{CDCl}_{3}$ unless otherwise stated. Mass spectra were recorded on Thermo-Fischer DSQ II GCMS instrument. IR spectra were recorded on a Perkin-Elmer FTIR RXI spectrometer as KBr pallets. Melting points were recorded in Thiele's tube using paraffin oil and are uncorrected. Kinetic experiments were run on ELICO LI127 pH meter.

\section{Comparison of propylene oxide and tetrahydrofuran as scavenger of $\mathrm{HI}$ :}

For the comparison of opening of propylene oxide and tetrahydrofuran as scavenger, the HI was synthesized by heating $\mathrm{NaI}(5.0 \mathrm{~g}, 0.033 \mathrm{~mol})$ and $\mathrm{H}_{3} \mathrm{PO}_{4}(3.7 \mathrm{~mL}, 0.05 \mathrm{~mol}, 1.5 \mathrm{eq}$.) in a round bottom flask. ${ }^{18 \mathrm{~b}}$ It was condensed in a dry round bottom flask. From the distilled sample of HI $(0.505 \mathrm{~g})$ was taken and diluted with distilled water to $25 \mathrm{~mL}$. For the comparison study two sets were prepared, one for propylene oxide and another for tetrahydrofuran. From this diluted solution ( $5 \mathrm{~mL}, 0.103 \mathrm{~g}$ of HI, $0.8 \mathrm{mmol})$ was taken for each set. In one set propylene oxide $(0.0935 \mathrm{~g}, 0.11$ $\mathrm{mL}, 1.6 \mathrm{mmol}, 2$ equiv) and distilled water was added to adjust the volume of the system to $15 \mathrm{~mL}$. For the second set, tetrahydrofuran $(0.1161 \mathrm{~g}, 0.13 \mathrm{~mL}, 1.6 \mathrm{mmol}, 2 \mathrm{eq}$.$) and distilled water was$ added to make the volume of the system to $15 \mathrm{~mL}$.

Both sets were stirred at the same speed continuously while the $\mathrm{pH}$ was measured using a glass electrode attached to a $\mathrm{pH}$ meter at the interval of $5 \mathrm{~min}$. Readings for the comparison are presented below in Table 2 and a graph was plotted ( $\mathrm{pH} v$ s time in min.) as shown in Figure 1. 
Table 2. Comparison of consumption of $\mathrm{HI}$ by propylene oxide and tetrahydrofuran

\begin{tabular}{ccc}
\hline Time $(\min )$ & HI \& propylene oxide $\mathrm{pH}$ & HI \& tetrahydrofuran $\mathrm{pH}$ \\
\hline 5 & 2.00 & 1.75 \\
15 & 2.32 & 1.86 \\
25 & 2.46 & 1.96 \\
35 & 2.66 & 2.04 \\
45 & 2.78 & 2.10 \\
55 & 2.82 & 2.17 \\
65 & 2.88 & 2.20 \\
75 & 2.96 & 2.23 \\
\hline
\end{tabular}

\section{4-Nitrostilbene (1a)}

Mizoroki-Heck Reaction (Method A): Preparation of Catalyst Solution

A solution of palladium acetate $(0.011 \mathrm{~g}, 0.00495 \mathrm{mmol}, 0.1 \mathrm{~mol} \%)$ and 1-( $\alpha$-1-piperidylbenzyl)-2naphthol (ligand A) $\left(0.0018 \mathrm{~g}, 0.0059 \mathrm{mmol}, 1.2\right.$ eq. of $\left.\mathrm{Pd}(\mathrm{OAc})_{2}\right)$ was prepared in $N, N$ dimethylacetamide $(5 \mathrm{~mL})$ under nitrogen atmosphere. The mixture was stirred at room temperature until a homogeneous solution was obtained. This catalyst solution was repeatedly purged by $\mathrm{N}_{2}$ prior to use.

A two neck r.b. flask was charged with $p$-bromonitrobenzene $(1.0 \mathrm{~g}, 4.95 \mathrm{mmol})$, dry potassium carbonate $(1.71 \mathrm{~g}, 12.37 \mathrm{mmol}, 2.5$ eq.), TBAB $(0.319 \mathrm{~g}, 0.99 \mathrm{mmol}, 20 \mathrm{~mol} \%)$ and $N, N-$ dimethylacetamide $(10-15 \mathrm{~mL})$. The solution was repeatedly purged with $\mathrm{N}_{2}$. Styrene $(0.773 \mathrm{~g}, 7.42$ mmol, 1.5 eq.) was added at $60{ }^{\circ} \mathrm{C}$ and the mixture was heated up to $100{ }^{\circ} \mathrm{C}$. When the temperature reached $100{ }^{\circ} \mathrm{C}$, the previously prepared Pd catalyst solution was added dropwise and the mixture was heated to $140^{\circ} \mathrm{C}$ for $48 \mathrm{~h}$. After the completion of the reaction, the mixture was poured in $6 \mathrm{~N}$ $\mathrm{HCl}$ and extracted with dichloromethane. The combined organic phase was washed with water, brine and dried over anhydrous sodium sulfate. The solvent was removed under reduced pressure and the crude product was purified by column chromatography on silica gel using petroleum ether as eluent to afford pale yellow solid $(0.623,77 \%)$, mp. $156^{\circ} \mathrm{C}$ (Lit. $\left.154-157^{\circ} \mathrm{C}\right){ }^{26}$

IR $v(\mathrm{KBr}) \mathrm{cm}^{-1}: 3077,1631,1589,1508,1338,1185,1105,969,873,833,765,693$.

${ }^{1} \mathrm{H}-\mathrm{NMR}\left(400 \mathrm{MHz}, \mathrm{CDCl}_{3}\right): \delta 8.21-8.24(\mathrm{td}, \mathrm{J}=9.32 \& 2.4 \mathrm{~Hz}, 2 \mathrm{H}), 7.62-7.65(\mathrm{td}, \mathrm{J}=9.32 \& 2.36$ $\mathrm{Hz}, 2 \mathrm{H}), 7.38-7.42(\mathrm{~m}, 2 \mathrm{H}), 7.55-7.57(\mathrm{~m}, 2 \mathrm{H}), 7.34-7.36(\mathrm{~m}, 1 \mathrm{H}), 7.25-7.3(\mathrm{~d}, \mathrm{~J}=16.32 \mathrm{~Hz}, 1 \mathrm{H})$, 7.12-7.17 (d, J = $16.32 \mathrm{~Hz}, 1 \mathrm{H})$,

MS (EI): m/z, (\%) 226 (15), 225 (100), 208 (6), 195 (6), 180 (7), 179 (43), 178 (99), 177 (19), 152 (25), 151 (10), 89 (13), 76 (6).

\section{3-Nitrophenanthrene (2a)}

\section{General procedure for photodehydrocyclization (Method B)}

A solution of 4-nitrostilbene 1a $(0.250 \mathrm{~g}, 1.11 \mathrm{mmol})$, iodine $(0.309 \mathrm{~g}, 1.22 \mathrm{mmol}, 1.1$ eq.), THF $(1.6 \mathrm{~g}, 1.8 \mathrm{~mL}, 22.2 \mathrm{mmol})$ and toluene $(1.2 \mathrm{~L})$ was irradiated using a 125W HMPV lamp for 36 $\mathrm{h} /$ monitored by tlc. After the reaction was over, the excess of iodine was removed by washing the solution with aqueous $\mathrm{Na}_{2} \mathrm{~S}_{2} \mathrm{O}_{3}$, followed by distilled water. The organic layer was concentrated 
under the reduced pressure. The pure product was isolated as a yellow solid by column chromatography over silica gel, further crystallized from light petroleum ether $(0.077 \mathrm{~g}, 31 \%)$, mp. $175-177^{\circ} \mathrm{C}$ (Lit. $\left.175-177^{\circ} \mathrm{C}\right) .^{27 \mathrm{a}}$

IR $v(\mathrm{KBr}) \mathrm{cm}^{-1}: 3433,1610,1535,1503,1336,1232,1199,1107,1035,892,844,804,735$.

${ }^{1} \mathrm{H}-\mathrm{NMR}\left(500 \mathrm{MHz}, \mathrm{CDCl}_{3}\right): \delta 9.62-9.62(\mathrm{~d}, \mathrm{~J}=2.5 \mathrm{~Hz}, 1 \mathrm{H}), 8.76-8.78(\mathrm{~d}, \mathrm{~J}=8.5 \mathrm{~Hz}, 1 \mathrm{H}), 8.39-$ $8.41(\mathrm{dd}, \mathrm{J}=9.0,2.0,1 \mathrm{H}), 8.01-8.03(\mathrm{~d}, \mathrm{~J}=8.5 \mathrm{~Hz}, 1 \mathrm{H}), 7.95-7.98(\mathrm{~m}, 2 \mathrm{H}), 7.81-7.83(\mathrm{~d}, \mathrm{~J}=9.0$, $1 \mathrm{H}), 7.78-7.79$ (ddd, J = 8.5, 7.0, 1.5 Hz, 1H), 7.22-7.74 (ddd, J = 8.0, 7.0, 1.0 Hz, 1H).

MS (EI): m/z, (\%) 224 (16), 223 (100), 193 (27), 177 (62), 176 (72), 167 (21), 165 (43), 151 (51), 150 (28), 149 (81), 88 (25), 81 (34), 57 (49).

\section{4-Bromo-4'-nitrostilbene (1b)}

\section{General procedure for Wittig Reaction (Method C)}

A solution of $\mathrm{Na}$ metal $(0.083 \mathrm{~g}, 3.63 \mathrm{mmol})$ in anhydrous methanol $(5 \mathrm{~mL})$ was added dropwise to a suspension of the (4-bromobenzyl)triphenylphosphonium bromide salt (1.685 g, $3.63 \mathrm{mmol})$ and $p$-nitrobenzaldehyde $(0.500 \mathrm{~g}, 3.63 \mathrm{mmol})$ in anhydrous methanol $(10 \mathrm{~mL})$ under $\mathrm{N}_{2}$ at room temperature. The color of the solution changed to yellow. After completion of the addition, the resulting solution was stirred for $24 \mathrm{~h}$ at room temperature, till no starting material was seen on tlc. The mixture was poured in cold water to separate the precipitate. The aqueous solution along with the precipitates was extracted with ethyl acetate and solvent layer was washed with water, brine and dried over sodium sulfate. The solvent was removed under reduced pressure and crude product was purified on silica gel column to afford mostly trans form of the title stilbene as yellow solid (0.915 g, 91\%), mp. $198-201{ }^{\circ} \mathrm{C}$ (lit. $\left.201-203{ }^{\circ} \mathrm{C}\right) .{ }^{27 b}$

IR $v(\mathrm{KBr}) \mathrm{cm}^{-1}:$ 3436, 1632, 1589, 1505, 1333, 1178, 1103, 1069, 1004, 968, 842, 811, 747.

${ }^{1} \mathrm{H}-\mathrm{NMR}\left(400 \mathrm{MHz}_{\mathrm{CDCl}}\right.$ ): $\delta 8.21-8.24(\mathrm{ddd}, \mathrm{J}=8.88,2.32,1.88 \mathrm{~Hz}, 2 \mathrm{H}), 7.61-7.65(\mathrm{ddd}, \mathrm{J}=$ 9.32, 2.32, $1.84 \mathrm{~Hz}, 2 \mathrm{H}), 7.51-7.54$ (ddd, J = 8.88, 2.28, $1.84 \mathrm{~Hz}, 2 \mathrm{H}$ ), 7.40-7.43 (ddd, J = 9.00, $2.20,1.90 \mathrm{~Hz}, 2 \mathrm{H}), 7.18-7.22(\mathrm{~d}, \mathrm{~J}=16.36 \mathrm{~Hz}, 1 \mathrm{H}), 7.11-7.15(\mathrm{~d}, \mathrm{~J}=16.36 \mathrm{~Hz}, 1 \mathrm{H})$.

MS (EI): m/z, (\%) 306(4), 305 (14), 304 (4), 303 (14), 279 (9), 178 (29), 177 (11), 167 (39), 166 (10), 150 (15), 149 (100), 113 (11), 76 (13), 71 (19).

3-Bromo-6-nitrophenanthrene (2b) was obtained in $68 \%$ yield $(0.169 \mathrm{~g})$ on a $0.82 \mathrm{mmol}$ scale by (method B). It was purified by column chromatography on silica gel using petroleum ether as eluent to afford pale yellow solid, mp. $234-235{ }^{\circ} \mathrm{C}$ (Lit. $\left.234-235{ }^{\circ} \mathrm{C}\right) .{ }^{17}$

IR $v(\mathrm{KBr}) \mathrm{cm}^{-1}:$ 3082, 1621, 1591, 1530, 1497, 1437, 1342, 1075, 1028, 845, 736, 588, 509.

${ }^{1} \mathrm{H}-\mathrm{NMR}\left(400 \mathrm{MHz}, \mathrm{CDCl}_{3}\right): \delta 9.52(\mathrm{~d}, \mathrm{~J}=2.40 \mathrm{~Hz}, 1 \mathrm{H}), 8.89(\mathrm{~d}, \mathrm{~J}=2.20 \mathrm{~Hz}, 1 \mathrm{H}), 8.41-8.44(\mathrm{dd}, \mathrm{J}$ $=8.80,2.20 \mathrm{~Hz}, 1 \mathrm{H}), 8.02-8.04(\mathrm{~d}, \mathrm{~J}=8.80 \mathrm{~Hz}, 1 \mathrm{H}), 7.90-7.92(\mathrm{~d}, \mathrm{~J}=8.80 \mathrm{~Hz}, 1 \mathrm{H}), 7.82-7.84(\mathrm{~d}, \mathrm{~J}$ $=8.80 \mathrm{~Hz}, 1 \mathrm{H}), 7.81-7.83(\mathrm{~m}, 2 \mathrm{H})$.

MS (EI): $m / z,(\%)=304$ (3), 303 (10), 302 (4), 301 (12), 176 (21), 175 (8), 149 (15), 137 (17), 136 (17), 121 (18), 109 (17), 95 (27), 69 (100).

2-[2-(4 Nitrophenyl)ethenyl]naphthalene (3) was obtained in $90 \%$ yield $(0.411 \mathrm{~g})$ on a $1.65 \mathrm{mmol}$ scale by (method C). It was purified by column chromatography on silica gel to afford mostly trans form of the olefin as yellow solid, mp. $169-171^{\circ} \mathrm{C}$ (Lit. $\left.168-170{ }^{\circ} \mathrm{C}\right) .^{20}$

IR $v(\mathrm{KBr}) \mathrm{cm}^{-1}:$ 3431, 2922, 1625, 1589, 1509, 1335, 1179, 1105, 965, 861, 831, 745, 688. 
${ }^{1} \mathrm{H}-\mathrm{NMR}\left(400 \mathrm{MHz}, \mathrm{CDCl}_{3}\right): \delta 8.22-8.24(\mathrm{~d}, \mathrm{~J}=8.72 \mathrm{~Hz}, 2 \mathrm{H}), 7.87-7.91(\mathrm{~d}, \mathrm{~J}=16.40 \mathrm{~Hz}, 1 \mathrm{H})$, 7.82-7.84 (m, 3H), 7.73-7.76 (dd, J = 8.60, $1.24 \mathrm{~Hz}, 1 \mathrm{H}), 7.65-7.67(\mathrm{~d}, \mathrm{~J}=8.36,2 \mathrm{H}), 7.47-7.53(\mathrm{~m}$, $2 \mathrm{H}), 7.40-7.44(\mathrm{~d}, \mathrm{~J}=16.30 \mathrm{~Hz}, 1 \mathrm{H}), 7.23-7.25(\mathrm{~d}, \mathrm{~J}=8.32 \mathrm{~Hz}, 1 \mathrm{H})$.

MS (EI): $\mathrm{m} / z$, (\%) 276(20), 275 (100), 229 (33), 228 (85), 227 (33), 226 (36), 202 (24), 114 (26), 113 (29), 101 (21), 83 (12), 81 (11).

2-Nitrobenzo[c]phenanthrene (4) was obtained in $83 \%$ yield $(0.165 \mathrm{~g})$ on a $0.91 \mathrm{mmol}$ scale by (method B). It was purified by column chromatography on silica gel using petroleum ether as eluent to afford yellow solid, mp. $150-152^{\circ} \mathrm{C}$ (Lit. $150-152{ }^{\circ} \mathrm{C}$ ). ${ }^{17}$

IR $v(\mathrm{KBr}) \mathrm{cm}^{-1}:$ 3052, 3006, 2924, 1601, 1530, 1510, 1378, 1334, 845, 828, 800, 759, 736.

${ }^{1} \mathrm{H}-\mathrm{NMR}\left(400 \mathrm{MHz}, \mathrm{CDCl}_{3}\right): \delta 9.99-10.00(\mathrm{~d}, \mathrm{~J}=2.00 \mathrm{~Hz}, 1 \mathrm{H}), 8.95-8.97(\mathrm{~d}, \mathrm{~J}=8.50 \mathrm{~Hz}, 1 \mathrm{H})$, 8.35-8.38 (dd, J = 8.80, $2.20 \mathrm{~Hz}, 1 \mathrm{H}), 8.06-8.09(\mathrm{~d}, \mathrm{~J}=8.90 \mathrm{~Hz}, 1 \mathrm{H}), 8.04-8.06(\mathrm{dd}, \mathrm{J}=8.70,2.40$ $\mathrm{Hz}, 1 \mathrm{H}), 7.98-8.0(\mathrm{~d}, \mathrm{~J}=8.50 \mathrm{~Hz}, 1 \mathrm{H}), 7.96-7.98(\mathrm{~d}, \mathrm{~J}=7.80 \mathrm{~Hz}, 1 \mathrm{H}), 7.91-7.93(\mathrm{~d}, \mathrm{~J}=8.50 \mathrm{~Hz}$, $1 \mathrm{H}), 7.81-7.84(\mathrm{~d}, \mathrm{~J}=8.50 \mathrm{~Hz}, 1 \mathrm{H}), 7.77-7.81(\mathrm{~m}, 1 \mathrm{H}), 7.68-7.72(\mathrm{~m}, 1 \mathrm{H})$.

MS (EI): $\mathrm{m} / z$, (\%) 275 (11), 274 (60), 273 (95), 264 (25), 257 (13), 227 (18), 226 (53), 225 (13), 219 (82), 131 (37), 100 (19).

1,2-Bis(2-naphthyl)ethylene (5) was obtained in $60 \%$ yield $(0.540 \mathrm{~g})$ on a $3.52 \mathrm{mmol}$ scale by (method C). It was purified by column chromatography on silica gel to afford mostly trans form of the olefin as colorless solid, mp. $251-252^{\circ} \mathrm{C}$ (Lit. $\left.257-258^{\circ} \mathrm{C}\right) .^{28}$

IR $v(\mathrm{KBr}) \mathrm{cm}^{-1}: 3049,2361,1621,1504,1439,1362,960,896,862,818,747$.

MS (EI): m/z, (\%) 281 (23), 280 (100), 279 (72), 278 (33), 265 (17), 152 (14), 140 (34), 139 (43), 126 (20), 97 (23).

Benzo[ghi]perylene (7) was obtained in $43 \%$ yield $(0.106 \mathrm{~g})$ on a $0.89 \mathrm{mmol}$ scale by (method B). It was purified by column chromatography on silica gel using petroleum ether as eluent to afford pale yellow solid, mp. $265-270{ }^{\circ} \mathrm{C}\left(\right.$ Lit. $\left.270{ }^{\circ} \mathrm{C}\right) .{ }^{29}$

IR $v(\mathrm{KBr}) \mathrm{cm}^{-1}: 3044,2925,1612,1595,1510,1445,844,811,767,753$.

${ }^{1} \mathrm{H}-\mathrm{NMR}\left(400 \mathrm{MHz}, \mathrm{CDCl}_{3}\right): \delta 8.98-9.02(\mathrm{dd}, \mathrm{J}=7.80,0.80 \mathrm{~Hz}, 2 \mathrm{H}), 8.16-8.21(\mathrm{dd}, \mathrm{J}=7.70,0.80$ $\mathrm{Hz}, 2 \mathrm{H}), 8.34$ (s, 2H), 8.11-8.15 (d, J = 8.90 Hz, 2H), 8.05-8.09 (d, J = 8.80 Hz, 2H), 7.97-8.05 (d, J $=7.80 \mathrm{~Hz}, 2 \mathrm{H})$.

MS (EI): m/z, (\%) 278 (3), 277 (25), 276 (100), 275 (14), 274 (23), 138 (58), 137 (44), 136 (19), $125(8), 124(7)$.

2-[2-(4-Bromophenyl)ethenyl]naphthalene (10) was obtained in $76 \%$ yield $(1.575 \mathrm{~g})$ on a 6.7 mmol scale by (method C). It was purified by column chromatography on silica gel to afford mostly trans form of the olefin as colorless solid, mp. $192^{\circ} \mathrm{C}$. (Lit. 188.5-189 ${ }^{\circ} \mathrm{C}$ ).$^{30}$

IR $v(\mathrm{KBr}) \mathrm{cm}^{-1}: 3049,1583,1483,1396,1072,965,853,820,741$.

${ }^{1} \mathrm{H}-\mathrm{NMR}\left(400 \mathrm{MHz}, \mathrm{CDCl}_{3}\right): \delta$ 7.80-7.85 (m, 4H), 7.70-7.73 (dd, J = 8.64, $\left.1.68 \mathrm{~Hz}, 1 \mathrm{H}\right), 7.41-7.51$ (m, 6H), 7.24-7.28 (d, J = 16.32 Hz, 1H), 7.13-7.17 (d, J = 16.32 Hz, 1H).

MS (EI): m/z, (\%) 311 (13), 310 (61), 309 (15), 308 (70), 230 (15), 229 (89), 228 (100), 226 (33), 202 (15), 152 (06), 128 (07), 114 (57), 101 (26).

2-Bromobenzo $[c]$ phenanthrene (11) was obtained in $96 \%$ yield $(0.953 \mathrm{~g})$ on a $3.2 \mathrm{mmol}$ scale by (method B). It was purified by column chromatography on silica gel using petroleum ether as eluent to afford colorless solid, mp. $87-88{ }^{\circ} \mathrm{C}$ (Lit. 87-88 ${ }^{\circ} \mathrm{C}$ ). ${ }^{30,31}$ 
IR $v(\mathrm{KBr}) \mathrm{cm}^{-1}:$ 3044, 1600, 1588, 1485, 1440, 1109, 1082, 1039, 839, 781, 746, 599, 570, 529.

${ }^{1} \mathrm{H}-\mathrm{NMR}\left(400 \mathrm{MHz}, \mathrm{CDCl}_{3}\right): \delta 9.03-9.05(\mathrm{~d}, \mathrm{~J}=8.50 \mathrm{~Hz}, 1 \mathrm{H}), 9.48(\mathrm{~s}, 1 \mathrm{H}), 8.02-8.04(\mathrm{~d}, \mathrm{~J}=7.90$ $\mathrm{Hz}, 1 \mathrm{H}), 7.91-7.93(\mathrm{~d}, \mathrm{~J}=8.60 \mathrm{~Hz}, 1 \mathrm{H}), 7.87-7.90(\mathrm{~d}, \mathrm{~J}=8.60 \mathrm{~Hz}, 1 \mathrm{H}), 7.82-7.85$ (d, J = 8.50 Hz, $1 \mathrm{H}), 7.80-7.82(\mathrm{~d}, \mathrm{~J}=8.40 \mathrm{~Hz}, 1 \mathrm{H}), 7.74-7.76(\mathrm{~d}, \mathrm{~J}=7.20 \mathrm{~Hz}, 1 \mathrm{H}), 7.71-7.74(\mathrm{~d}, \mathrm{~J}=8.40 \mathrm{~Hz}, 1 \mathrm{H})$, 7.65-7.67 (d, J = 7.40 Hz, 1H), 7.63-7.65 (d, J = 7.30 Hz, 1H).

MS (EI): m/z, (\%) 308 (50), 307 (12), 306 (51), 227 (44), 226 (76), 225 (19), 224 (22), 149 (39), 114 (27), 113 (100), 112 (51), 111 (23), 97 (17).

\section{2-Styrylbenzo[c]phenanthrene (12)}

Mizoroki-Heck Reaction (Method D): Preparation of Catalyst Solution

A solution of palladium acetate $(0.0015 \mathrm{~g}, 0.0065 \mathrm{mmol}, 1 \mathrm{~mol} \%)$ and 1,3bis(diphenylphospino)propane $\left(0.004 \mathrm{~g}, 0.0097 \mathrm{mmol}, 1.5\right.$ eq. of $\left.\mathrm{Pd}(\mathrm{OAc})_{2}\right)$ was prepared in $N, N-$ dimethylacetamide $(5 \mathrm{~mL})$ under nitrogen atmosphere. The mixture was stirred at room temperature until a homogeneous solution was obtained. This solution was repeatedly purged by $\mathrm{N}_{2}$ prior to use. In another r.b. flask, a mixture of 2-bromobenzo[c]phenanthrene 11 (0.2 g, $0.65 \mathrm{mmol})$, dry potassium carbonate $(0.180 \mathrm{~g}, 1.3 \mathrm{mmol}, 2 \mathrm{eq}$.$) , TBAB (0.104 \mathrm{~g}, 0.13 \mathrm{mmol}, 20 \mathrm{~mol} \%)$ was prepared in dry $\mathrm{N}, \mathrm{N}$-dimethylacetamide $(15 \mathrm{~mL})$ and degassed. This was heated to $60{ }^{\circ} \mathrm{C}$ and charged with styrene $\left(0.102 \mathrm{~g}, 0.97 \mathrm{mmol}, 1.5 \mathrm{eq}\right.$.) and the mixture was heated up to $100{ }^{\circ} \mathrm{C}$. When the temperature reached $100{ }^{\circ} \mathrm{C}$, the previously prepared $\mathrm{Pd}$ catalyst solution was added dropwise and the mixture was heated to $140{ }^{\circ} \mathrm{C}$ for $48 \mathrm{~h}$. After completion of the reaction, the mixture was poured in $6 \mathrm{~N} \mathrm{HCl}$ and extracted with dichloromethane. The combined organic phase was washed with water, brine and dried over sodium sulfate. The solvent was removed under reduced pressure and the crude product was purified by column chromatography on silica gel to get pure colorless solid product $(0.210 \mathrm{~g}, 98 \%)$, mp. $140-42{ }^{\circ} \mathrm{C}$ (Lit. $\left.140{ }^{\circ} \mathrm{C}\right) .{ }^{32}$

IR $v(\mathrm{KBr}) \mathrm{cm}^{-1}: 3018,1601,1497,1445,957,832,783,745,687$.

$\left.{ }^{1} \mathrm{H}-\mathrm{NMR}\left(400 \mathrm{MHz}, \mathrm{CDCl}_{3}\right): \delta 9.18-9.20 \mathrm{~d}, \mathrm{~J}=7.20 \mathrm{~Hz}, 1 \mathrm{H}\right), 9.18(\mathrm{~s}, 1 \mathrm{H}), 8.04-8.07$ (m, 2H), 7.89$7.94(\mathrm{~m}, 2 \mathrm{H}), 7.81-7.86(\mathrm{~m}, 2 \mathrm{H}), 7.43-7.78(\mathrm{~m}, 1 \mathrm{H}), 7.63-7.69(\mathrm{~m}, 3 \mathrm{H}), 7.41-7.45$ (m, 3H), 7.28$7.35(\mathrm{~m}, 3 \mathrm{H})$.

MS (EI): m/z, (\%) 331(26), 330 (100), 329 (29), 328 (15), 313 (10), 226 (8), 165 (30), 164 (22), 163 (21), 157 (25), 156 (18), 151 (8).

2-[2-(4-Methylphenyl)ethenyl]benzo[c]phenanthrene (13) was obtained in $95 \%$ yield $(0.531 \mathrm{~g})$, on a 1.62-mmol scale, by (method D). It was purified by column chromatography on silica gel to afford pale yellow solid, mp. $115-116{ }^{\circ} \mathrm{C}$ (Lit. $\left.118-119{ }^{\circ} \mathrm{C}\right) .{ }^{33}$

IR $v(\mathrm{KBr}) \mathrm{cm}^{-1}: 3023,2915,1598,1508,1414,1230,1181,961,838,802,747,671,610$.

${ }^{1} \mathrm{H}-\mathrm{NMR}\left(400 \mathrm{MHz}, \mathrm{CDCl}_{3}\right): \delta 9.14-9.16(\mathrm{~d}, \mathrm{~J}=8.56 \mathrm{~Hz}, 1 \mathrm{H}), 9.12(\mathrm{~s}, 1 \mathrm{H}), 8.01-8.03(\mathrm{dd}, \mathrm{J}=8.00$, $1.32 \mathrm{~Hz}, 1 \mathrm{H}), 7.96-7.98(\mathrm{~d}, \mathrm{~J}=8.36 \mathrm{~Hz}, 1 \mathrm{H}), 7.84-7.90(\mathrm{~m}, 3 \mathrm{H}), 7.79-7.81(\mathrm{~d}, \mathrm{~J}=8.56 \mathrm{~Hz}, 1 \mathrm{H})$, 7.76-7.79 (d, J = 8.48 Hz, 2H), 7.70-7.74 (ddd, J = 8.48, 6.96, $1.48 \mathrm{~Hz}, 1 \mathrm{H}), 7.61-7.65$ (ddd, $\mathrm{J}=$ 8.00, 7.00, $1.08 \mathrm{~Hz}, 1 \mathrm{H}), 7.48-7.50$ (d, J = 8.04 Hz, 2H), 7.32-7.36 (d, J = 16.36 Hz, 1H), 7.24-7.28 $(\mathrm{d}, \mathrm{J}=16.36 \mathrm{~Hz}, 1 \mathrm{H}), 7.19-7.21(\mathrm{~d}, \mathrm{~J}=7.88 \mathrm{~Hz}, 1 \mathrm{H}), 2.37$ (s, 3H).

MS (EI): m/z, (\%) 345(29), 344 (100), 329 (15), 328 (19), 226 (07), 165 (22), 164 (34), 156 (13). 
[6]Helicene (14) was obtained in $64 \%$ yield $(0.083 \mathrm{~g})$ on a $0.39 \mathrm{mmol}$ scale by (method B). It was purified by column chromatography on silica gel using petroleum ether as eluent to afford yellow solid, mp 231-232 ${ }^{\circ} \mathrm{C}$. (Lit. $231^{\circ} \mathrm{C}$ ). ${ }^{33}$

Same compound was also synthesized by double photodehydrocyclization of 18 (Scheme-6).

A solution of 2,7-distyrylnaphthalene $18(0.110 \mathrm{~g}, 0.38 \mathrm{mmol})$, iodine (0.214 g, $0.84 \mathrm{mmol} 2.2$ eq.), tetrahydrofuran $(1.1 \mathrm{~g}, 1.23 \mathrm{~mL}, 15.2 \mathrm{mmol}, 40 \mathrm{eq}$.$) and toluene (1.2 \mathrm{~L})$ was irradiated using $125 \mathrm{~W}$ HMPV lamp for $22 \mathrm{~h}$ and was monitored by tlc. After the reaction was over, the excess of iodine was removed by washing the solution with aqueous $\mathrm{Na}_{2} \mathrm{~S}_{2} \mathrm{O}_{3}$, followed by distilled water. The organic layer was concentrated at reduced pressure. The pure product was obtained after column chromatography as yellow solid $(0.081 \mathrm{~g}, 75 \%)$ (recrystallized from petroleum ether).

mp. $231-232^{\circ} \mathrm{C}$ (Lit. $\left.231^{\circ} \mathrm{C}\right) .{ }^{33}$

IR $v(\mathrm{KBr}) \mathrm{cm}^{-1}: 3042,1601,1500,1468,1434,844,828,799,753$.

${ }^{1} \mathrm{H}-\mathrm{NMR}\left(400 \mathrm{MHz}, \mathrm{CDCl}_{3}\right): \delta$ 7.96-8.01 (m, 4H), 7.90-7.95 (m, 4H), 7.83-7.88 (d, J = 8.00 Hz, $2 \mathrm{H}), 7.57-7.59(\mathrm{~d}, \mathrm{~J}=8.60 \mathrm{~Hz}, 2 \mathrm{H}), 7.19-7.23(\mathrm{dd}, \mathrm{J}=7.70,7.20 \mathrm{~Hz}, 2 \mathrm{H}), 6.65-6.69(\mathrm{~m}, 2 \mathrm{H})$.

MS (EI): m/z, (\%) 329 (26), 328 (100), 301 (43), 300 (69), 163 (49), 162 (60), 161 (27), 156 (50), 150 (48), 149 (45), 111 (32), 97 (49), 85 (61), 83 (61).

2-Methyl[6]helicene (15) was obtained in $78 \%$ yield $(0.193 \mathrm{~g}$ ) on a $0.75 \mathrm{mmol}$ scale by (method B). It was purified by column chromatography on silica gel using petroleum ether as eluent to afford pale yellow solid, recrystallized from ethyl acetate-petroleum ether. mp. 194-196 ${ }^{\circ} \mathrm{C}$ (Lit. 194-198 $\left.{ }^{\circ} \mathrm{C}\right) .{ }^{34}$

IR $v(\mathrm{KBr}) \mathrm{cm}^{-1}: 3045,2911,1603,1504,1475,1438,1144,1036,951,848,833,794,747,733$, 618.

${ }^{1} \mathrm{H}-\mathrm{NMR}\left(400 \mathrm{MHz}, \mathrm{CDCl}_{3}\right): \delta$ 7.92-8.02 (m, 6H), 7.82-7.88 (m, 2H), 7.79-7.81 (dd, J = 8.04, 0.80 $\mathrm{Hz}, 1 \mathrm{H}), 7.69-7.71(\mathrm{~d}, \mathrm{~J}=8.08 \mathrm{~Hz}, 1 \mathrm{H}), 7.58-7.60(\mathrm{~d}, \mathrm{~J}=8.48, \mathrm{~Hz}, 1 \mathrm{H}), 7.36(\mathrm{~S}, 1 \mathrm{H}), 7.21-7.23$ $(\mathrm{dd}, \mathrm{J}=7.88,0.88 \mathrm{~Hz}, 1 \mathrm{H}), 7.03-7.05(\mathrm{dd}, \mathrm{J}=8.08,1.32 \mathrm{~Hz}, 1 \mathrm{H}), 6.65-6.69$ (ddd, $\mathrm{J}=8.36,6.92$, $1.32 \mathrm{~Hz}, 1 \mathrm{H}), 1.75(\mathrm{~S}, 3 \mathrm{H})$.

MS (EI): m/z, (\%) 343 (30), 342 (100), 327 (49), 326 (35), 300 (45), 171 (14), 163 (60), 162 (62), 150 (28).

2,7-Dibromonaphthalene (17). A $250 \mathrm{~mL}$ round bottom flask was charged with triphenylphosphine (14.41g, $54.9 \mathrm{mmol}, 2.2$ eq.) and dry acetonitrile (20 mL). The suspension was cooled to $0{ }^{\circ} \mathrm{C}$ and to this mixture dry bromine $(8.77 \mathrm{~g}, 2.8 \mathrm{~mL}, 54.93 \mathrm{mmol}, 2.2 \mathrm{eq}$.) was added dropwise in such a way that the temperature was maintained below $5{ }^{\circ} \mathrm{C}$. A heavy white precipitate was formed. To this mixture 2,7-dihydroxynaphthalene (16) in acetonitrile $(40 \mathrm{~mL})$ was added in one portion. The mixture was carefully stirred even though precipitates gained weight. The mixture was heated to $70-80{ }^{\circ} \mathrm{C}$ at which point the precipitate dissolved and the reaction continued for $1.5 \mathrm{~h}$. The flask was fitted with an air cooled distillation assembly and acetonitrile was distilled at atmospheric pressure. The temperature was then gradually increased up to $250^{\circ} \mathrm{C}$. Evolution of $\mathrm{HBr}$ appeared with strong heating $\left(\sim 190^{\circ} \mathrm{C}\right)$. This evolved $\mathrm{HBr}$ was trapped by a saturated $\mathrm{NaOH}$ solution. The temperature was maintained around $280-300{ }^{\circ} \mathrm{C}$ for $2 \mathrm{~h}$ and a dark oil was obtained which was allowed to cool to $100{ }^{\circ} \mathrm{C}$ at which point $50 \mathrm{~mL}$ of absolute ethanol was added and the mixture stored at $4{ }^{\circ} \mathrm{C}$ overnight. The product was extracted with petroleum ether, and separated by 
column chromatography ( $\mathrm{SiO}_{2}$, Petroleum ether) to afford a colorless solid (3.76 g, 53\%), which was recrystallized from ethanol for further purification.

mp. $138-140{ }^{\circ} \mathrm{C}$ (Lit. $\left.140-141^{\circ} \mathrm{C}\right) .{ }^{35}$

IR $v(\mathrm{KBr}) \mathrm{cm}^{-1}: 3052,1606,1567,1482,1344,1178,933,903,867,836,636,592,538,473$.

MS (EI): $m / z$ (\%) 288 (42), 286 (100), 284 (46), 207 (21), 205 (23), 144 (09), 143 (18), 142 (09), 126 (84), 125 (10), 103 (08), 74 (11), 63 (38).

2,7-Distyrylnaphthalene (18) was obtained in $65 \%$ yield $(0.377 \mathrm{~g})$ on a $1.74 \mathrm{mmol}$ scale by (method D). It was purified by column chromatography on silica gel to afford colorless solid, mp. 262-264 ${ }^{\circ} \mathrm{C}$ (Lit. $\left.260-262{ }^{\circ} \mathrm{C}\right) .{ }^{36}$

IR $v(\mathrm{KBr}) \mathrm{cm}^{-1}: 3053,3022,1619,1492,1445,966,913,836,747,691$.

${ }^{1} \mathrm{H}-\mathrm{NMR}$ (400 MHz, d ${ }^{6}$-DMSO): $\delta$ 7.92-7.98 (m, 12H including 1 olefin $\mathrm{H}$ ), 7.79-7.83 (m, 2H, for olefin $\mathrm{J}=17.6 \mathrm{~Hz}), 7.60-7.62(\mathrm{~m}, 2 \mathrm{H}), 7.38-7.41(\mathrm{~m}, 2 \mathrm{H}), 7.28-7.32(\mathrm{~m}, 2 \mathrm{H})$.

MS (EI): $m / z,(\%) 333$ (27) 332 (100), 254 (7), 253 (26), 229 (11), 228 (20), 226 (09), 166 (12), 164 (9), $158(26), 151$ (13).

2-[2-(4-Methylphenyl)ethenyl]naphthalene (21) was obtained in 86\% yield (3.552 g) on a 16.96 mmol scale by (method C). It was purified by column chromatography on silica gel to afford mostly trans form of the olefin as colorless solid, mp. $183-184^{\circ} \mathrm{C}$ (Lit. $\left.181-183^{\circ} \mathrm{C}\right) .^{37}$

IR $v(\mathrm{KBr}) \mathrm{cm}^{-1}:$ 3016, 2913, 2361, 1907, 1591, 1508, 1411, 1363, 1274, 1112, 965, 901, 853, 821, 740.

MS (EI): m/z, (\%) 245 (21), 244 (100), 243 (23), 230 (18), 229 (85), 228 (60), 167 (28), 148 (69), 129 (16), 122 (15), 121 (22), 114 (22), 113 (23), 101 (15), 83 (20), 71 (20).

2-Methylbenzo[c]phenanthrene (22) was obtained in $96 \%$ yield $(0.236 \mathrm{~g})$ on a $1.02 \mathrm{mmol}$ scale by (method B). It was purified by column chromatography on silica gel using petroleum ether as eluent to afford colorless solid, mp. $79-81{ }^{\circ} \mathrm{C}$ (Lit. $\left.79-80{ }^{\circ} \mathrm{C}\right) .{ }^{37}$

IR $v(\mathrm{KBr}) \mathrm{cm}^{-1}:$ 3047, 3010, 2919, 1598, 1518, 1493, 1417, 838, 785, 754, 669.

${ }^{1} \mathrm{H}-\mathrm{NMR}\left(400 \mathrm{MHz}, \mathrm{CDCl}_{3}\right): \delta 9.13-9.15(\mathrm{~d}, \mathrm{~J}=8.44 \mathrm{~Hz}, 1 \mathrm{H}), 8.93-8.93(\mathrm{~d}, \mathrm{~J}=0.64 \mathrm{~Hz}, 1 \mathrm{H})$, 7.99- $8.02(\mathrm{dd}, \mathrm{J}=7.96,1.40 \mathrm{~Hz}, 1 \mathrm{H}), 7.90-7.92(\mathrm{~d}, \mathrm{~J}=8.12 \mathrm{~Hz}, 1 \mathrm{H}), 7.84-7.88(\mathrm{t}, \mathrm{J}=8.28 \mathrm{~Hz}$, 2H), 7.79-7.81 (d, J = 8.52 Hz, 1H), 7.74- $7.76(\mathrm{~d}, \mathrm{~J}=8.48 \mathrm{~Hz}, 1 \mathrm{H}), 7.66-7.70(\mathrm{ddd}, \mathrm{J}=8.40,6.84$, $1.52 \mathrm{~Hz}, 1 \mathrm{H}), 7.59-7.63(\mathrm{ddd}, \mathrm{J}=8.00,7.00,1.16 \mathrm{~Hz}, 1 \mathrm{H}), 7.44-7.47(\mathrm{dd}, \mathrm{J}=8.08,1.32 \mathrm{~Hz}, 1 \mathrm{H})$, $2.65(\mathrm{~S}, 3 \mathrm{H})$.

MS (EI): m/z, (\%) 243 (22), 242 (100), 241 (17), 227 (29), 226 (31), 167 (8), 149 (27), 121 (12), 120 (15), 119 (31), 113 (25), 106 (10), 81 (8).

\section{2-Benzo[c]phenanthrylmethyltriphenylphosphonium bromide (23)}

A solution of 2-methylbenzo[c]phenanthrene $22(0.250 \mathrm{~g}, 1.03 \mathrm{mmol}), \mathrm{N}$-bromosuccinimide $(0.184$

$\mathrm{g}, 1.03 \mathrm{mmol})$ and benzoyl peroxide $(0.025 \mathrm{~g}, 0.1 \mathrm{mmol})$ in carbon tetrachloride $(10 \mathrm{~mL})$ was heated to reflux and stirred for $8 \mathrm{~h}$. At the end of this time the reaction mixture was cooled and the succinimide produced was removed by filtration. The filtrate was washed with a sodium thiosulphate solution, water and was dried over sodium sulfate. The solvent was then removed under reduced pressure. 
The crude product $(0.330 \mathrm{~g})$ and triphenylphosphine $(0.295 \mathrm{~g}, 1.12 \mathrm{mmol})$ was dissolved in xylene and refluxed with stirring for $8 \mathrm{~h}$. The precipitates formed were collected, washed with petroleum ether and dried in vacuum affording a white solid (0.61 g, 98\%), mp. 298-302 ${ }^{\circ} \mathrm{C}$. (Lit. mp. 308 $\left.{ }^{\circ} \mathrm{C}\right) .{ }^{37}$

2-[2-(Naphthyl)ethenyl]benzo[c]phenanthrene (24) was obtained in $54 \%$ yield $(0.660 \mathrm{~g})$ on a 3.2 mmol scale by (method C). It was purified by column chromatography on silica gel to afford mostly trans form of the olefin as colorless solid, mp. 198-200 ${ }^{\circ} \mathrm{C}$ (Lit. 200-201 ${ }^{\circ} \mathrm{C}$ ). ${ }^{23}$

IR $v(\mathrm{KBr}) \mathrm{cm}^{-1}:$ 3046, 2364, 1919, 1595, 1500, 1426, 1237, 959,895, 820, 811, 744, 621.

${ }^{1} \mathrm{H}-\mathrm{NMR}\left(500 \mathrm{MHz}, \mathrm{CDCl}_{3}\right): \delta 9.18-9.20(\mathrm{~d}, \mathrm{~J}=8.50 \mathrm{~Hz}, 1 \mathrm{H}), 9.18(\mathrm{~s}, 1 \mathrm{H}), 8.02-8.06(\mathrm{~m}, 2 \mathrm{H})$, 7.81-7.85 (m, $10 \mathrm{H}), 7.75-7.78(\mathrm{~m}, 1 \mathrm{H}), 7.65-7.68(\mathrm{~m}, 1 \mathrm{H}), 7.45-7.55(\mathrm{~m}, 4 \mathrm{H}, 2$-aromatic \& 2olefinic protons, $\mathrm{J}=16.50 \mathrm{~Hz}$ for olefinic $\mathrm{H})$.

MS (EI): m/z, (\%) 381 (31), 380 (100), 379 (36), 378 (16), 363 (11), 226 (7), 190 (37), 189 (38), 188 (27), $182(26), 176(6)$.

Dinaphtho[1,2-a:2',1'- $\boldsymbol{h}$ ] anthracene (25) was obtained in 75\% yield $(0.093 \mathrm{~g})$ on a $1.02 \mathrm{mmol}$ scale by (method B). It was purified by column chromatography on silica gel using petroleum ether as eluent to afford yellow solid, mp. $230-231{ }^{\circ} \mathrm{C}$ (Lit. $230-231{ }^{\circ} \mathrm{C}$ ). ${ }^{23}$

IR $v(\mathrm{KBr}) \mathrm{cm}^{-1}: 3047,1596,1501,1419,1387,1223,902,841,797,753$.

${ }^{1} \mathrm{H}-\mathrm{NMR}\left(400 \mathrm{MHz}, \mathrm{CDCl}_{3}\right): \delta 9.66(\mathrm{~S}, 2 \mathrm{H}), 9.36-9.38(\mathrm{~d}, \mathrm{~J}=8.52,2 \mathrm{H}), 8.09-8.12(\mathrm{~m}, 4 \mathrm{H}), 8.00-$ $8.02(\mathrm{~d}, \mathrm{~J}=8.48 \mathrm{~Hz}, 2 \mathrm{H}), 7.86-7.92(\mathrm{dd}, \mathrm{J}=8.68,8.48 \mathrm{~Hz}, 4 \mathrm{H}), 7.78-7.82$ (ddd, J = 6.92, 1.48, 1.40 $\mathrm{Hz}, 2 \mathrm{H}), 7.67-7.71$ (ddd, J = 7.92, 1.00, $0.96 \mathrm{~Hz}, 2 \mathrm{H})$.

MS (EI): m/z, (\%) 379 (30), 378 (91), 376 (22), 256 (16), 189 (48), 188 (44), 187 (48), 129 (34), 111 (49), 98 (58), 97 (81), 85 (90), 83 (100), 82 (49), 71 (98).

4,4'-Dibromostilbene (28) was obtained in $91 \%$ yield $(0.826 \mathrm{~g})$ on a $2.7 \mathrm{mmol}$ scale by (method C). It was purified by column chromatography on silica gel to afford mostly trans form of the olefin as colorless solid, mp. $210-212{ }^{\circ} \mathrm{C}$ (Lit. $\left.215-6{ }^{\circ} \mathrm{C}\right) .{ }^{25 \mathrm{~d}}$

IR $v(\mathrm{KBr}) \mathrm{cm}^{-1}: 3049,1901,1721,1584,1494,1405,1379,1230,1151,1104,1070,1018,837$, 769, 695, 607, 575, 517.

${ }^{1} \mathrm{H}-\mathrm{NMR}\left(400 \mathrm{MHz}, \mathrm{CDCl}_{3}\right): \delta$ 7.46-7.50 (d, J = 8.55 Hz, 4H), 7.35-7.38 (d, J = 8.55 Hz, 4H), 7.02 $(\mathrm{s}, 2 \mathrm{H})$.

MS (EI): m/z, (\%) 340 (23), 339 (8), 338 (47), 336(27), 179 (16), 178 (100), 177 (16), 176 (21), 152 (10), 151 (10), 89 (48), 88 (33).

3,6-Dibromophenanthrene (29) was obtained in $97 \%$ yield $(0.241 \mathrm{~g})$ on a $0.81 \mathrm{mmol}$ scale by (method B). It was purified by column chromatography on silica gel using petroleum ether as eluent to afford colorless solid, mp. $194^{\circ} \mathrm{C}$ (Lit.- $\left.194^{\circ} \mathrm{C}\right) .{ }^{25 \mathrm{~d}}$

IR $v(\mathrm{KBr}) \mathrm{cm}^{-1}:$ 3049, 2918, 1583, 1494, 1405, 1379, 1069, 1017, 948, 837, 769, 732, 606, 517.

${ }^{1} \mathrm{H}-\mathrm{NMR}\left(400 \mathrm{MHz}, \mathrm{CDCl}_{3}\right): \delta 8.75-8.76(\mathrm{~d}, \mathrm{~J}=1.56 \mathrm{~Hz}, 2 \mathrm{H}), 7.80-7.83(\mathrm{~d}, \mathrm{~J}=8.48 \mathrm{~Hz}, 2 \mathrm{H}), 7.71-$ 7.75 (m, with overlapping $\mathrm{d}, \mathrm{J}=8.48 \mathrm{~Hz}, 4 \mathrm{H})$.

MS (EI): m/z, (\%) 338 (30), 336 (61), 334 (33), 177 (17), 176 (100), 175 (20), 174 (17), 168 (18), 167 (38), 150 (26), 149 (99), 88 (63), 87 (25), 71 (26). 
3,6-Distyrylphenanthrene (30) was obtained in $82 \%$ yield $(0.233 \mathrm{~g})$ on a $0.74 \mathrm{mmol}$ scale by (method D). It was purified by column chromatography on silica gel to afford colorless solid, 38 mp.198-200 ${ }^{\circ} \mathrm{C}$.

IR $v(\mathrm{KBr}) \mathrm{cm}^{-1}:$ 3022, 1802, 1601, 1494, 1446, 1154, 958, 886, 840, 788, 750, 688.

${ }^{1} \mathrm{H}-\mathrm{NMR}\left(500 \mathrm{MHz}, \mathrm{CDCl}_{3}\right): \delta 8.76(\mathrm{~s}, 2 \mathrm{H}), 7.85-7.89(\mathrm{~m}, 4 \mathrm{H}), 7.70(\mathrm{~s}, 2 \mathrm{H}), 7.63-7.64(\mathrm{~d}, \mathrm{~J}=7.0$ $\mathrm{Hz}, 4 \mathrm{H}), 7.40-7.45(\mathrm{~m}, 6 \mathrm{H}), 7.32-7.36(\mathrm{~d}, \mathrm{~J}=16.5 \mathrm{~Hz}, 2 \mathrm{H}), 7.29-7.31$ (d, J = 7.5 Hz, 2H).

MS (EI): $m / z,(\%)=384$ (4), 383 (32), 382 (100), 303 (13), 302 (9), 289 (8), 278 (8), 277 (6), 276 (10), 191 (19), 183 (10), 182 (17), 151 (13).

[7]Helicene (26) was obtained by method B. A solution of 3,6-distyrylphenanthrene $30(0.150 \mathrm{~g}$, $0.39 \mathrm{mmol})$, iodine (0.219 g, $8.64 \mathrm{mmol}, 2.2$ eq.), THF (1.32 g, $1.27 \mathrm{~mL}, 15.71 \mathrm{mmol}, 40$ eq.) and toluene $(1.2 \mathrm{~L})$ was irradiated using $125 \mathrm{~W}$ HMPV lamp for $9 \mathrm{~h}$. After the reaction was over the excess of iodine was removed by washing the solution with aqueous $\mathrm{Na}_{2} \mathrm{~S}_{2} \mathrm{O}_{3}$, followed by distilled water. The organic layer was concentrated at reduced pressure. The pure product as yellow solid was obtained after column chromatography (0.066 g, 44\%), mp. 254-256 ${ }^{\circ} \mathrm{C}$ (Lit. 254-255 ${ }^{\circ} \mathrm{C}$.). ${ }^{15 a}, 38$ IR $v(\mathrm{KBr}) \mathrm{cm}^{-1}: 2925,2364,1610,1049,839,608$.

${ }^{1} \mathrm{H}-\mathrm{NMR}\left(500 \mathrm{MHz}, \mathrm{CDCl}_{3}\right): \delta 8.03(\mathrm{~s}, 2 \mathrm{H}), 7.99-8.01(\mathrm{~d}, \mathrm{~J}=8.50 \mathrm{~Hz}, 2 \mathrm{H}), 7.92-7.93(\mathrm{~d}, \mathrm{~J}=8.00$ $\mathrm{Hz}, 2 \mathrm{H}), 7.73-7.75(\mathrm{~d}, \mathrm{~J}=8.50 \mathrm{~Hz}, 2 \mathrm{H}), 7.49-7.51(\mathrm{~d}, \mathrm{~J}=8.50 \mathrm{~Hz}, 2 \mathrm{H}), 7.29-7.31$ (d, J = 7.50 Hz, $2 \mathrm{H}), 7.15-7.17(\mathrm{~d}, \mathrm{~J}=8.50 \mathrm{~Hz}, 2 \mathrm{H}), 6.89-6.92(\mathrm{t}, \mathrm{J}=7.50 \mathrm{~Hz}, 2 \mathrm{H}), 6.38-6.41(\mathrm{t}, \mathrm{J}=7.00 \mathrm{~Hz}, 2 \mathrm{H})$.

MS (EI): $m / z,(\%)=378$ (31), 377 (12), 351 (10), 350 (8), 256 (18), 187 (26), 129 (31), 111 (46), 98 (59), 97 (76), 83 (96), 71 (100).

\section{3,6 -Dibromo-9,10-phenanthrenequinone (32)}

To a suspension of phenanthrene $31(3.0 \mathrm{~g}, 17 \mathrm{mmol})$ in dilute $\mathrm{H}_{2} \mathrm{SO}_{4}\left(30 \mathrm{~mL}\right.$, conc. $\mathrm{H}_{2} \mathrm{SO}_{4}$ in 60 $\mathrm{mL} \mathrm{H}_{2} \mathrm{O}$ ), $\mathrm{K}_{2} \mathrm{Cr}_{2} \mathrm{O}_{7}\left(18 \mathrm{~g}\right.$ ) was added at $90-95{ }^{\circ} \mathrm{C}$ (water bath) in small portions $(0.5-1 \mathrm{~g})$ until a vigorous reaction sets in. The external heating was removed and the temperature of the mixture was kept approximately $110-115^{\circ} \mathrm{C}$. The temperature of the reaction mixture was not allowed to drop below $85{ }^{\circ} \mathrm{C}$ (hot water bath can be used if necessary). Finally the mixture was heated on a boiling water bath for $30 \mathrm{~min}$, cooled, then water $(200 \mathrm{~mL})$ was added and the crude product was filtered, washed with water, purified by suspending in ethanol $(30 \mathrm{~mL})$ and stirred with saturated sodium bisulphate solution $(30 \mathrm{~mL})$. The mixture was stirred for $10 \mathrm{~min}$, diluted with water $(175 \mathrm{~mL})$ and filtered. The filtrate was treated with a saturated sodium carbonate solution. The precipitated 9,10phenanthrenequinone was filtered, washed with water, crystallized from glacial acetic acid, to obtain an orange solid. (4.046 g, 57.7\%), mp. 207-209 ${ }^{\circ} \mathrm{C}$ (Lit. 208.5-210 $\left.{ }^{\circ} \mathrm{C}\right) .{ }^{39 \mathrm{a}} \mathrm{IR} v(\mathrm{KBr}) / \mathrm{cm}^{-1}$ : $3067,1675,1593,1478,1451,1229,972,893,766,716$.

A mixture of 9,10-phenanthrenequinone $(0.5 \mathrm{~g}, 2.4 \mathrm{mmol})$, dry nitrobenzene $(3.0 \mathrm{~mL})$ and dry bromine (1.35 mL, $26.4 \mathrm{mmol}$, dried over conc. $\left.\mathrm{H}_{2} \mathrm{SO}_{4}\right)$ and benzoyl peroxide $(0.029 \mathrm{~g}, 0.12 \mathrm{mmol})$ was exposed to a coiled coil $200 \mathrm{~W}$ tungsten lamp made by Edison India. Within about $10 \mathrm{~min}$, evolution of gas bubbles could be seen. The temperature of the reaction was kept at $75{ }^{\circ} \mathrm{C}$. After about $1 \mathrm{hr}$, the evolution of gas became imperceptible and cluster of needles gradually separated. The crude product was filtered, dried and recrystallized from absolute ethanol to obtain orange needles $(0.50 \mathrm{~g}, 72.5 \%)$. mp. $258-260{ }^{\circ} \mathrm{C}$ (Lit. $\left.259-276^{\circ} \mathrm{C}\right) .{ }^{39 b}$ 
IR $v(\mathrm{KBr}) \mathrm{cm}^{-1}: 3101,3034,2921,2852,1677,1583,1545,1470,1225,967,903,828,701$.

${ }^{1} \mathrm{H}-\mathrm{NMR}\left(200 \mathrm{MHz}, \mathrm{CDCl}_{3}\right): \delta 8.11(\mathrm{~s}, 2 \mathrm{H}), 8.04-8.08(\mathrm{~d}, \mathrm{~J}=8.00 \mathrm{~Hz}, 2 \mathrm{H}), 7.64-7.68(\mathrm{~d}, \mathrm{~J}=8.00$ $\mathrm{Hz}, 2 \mathrm{H})$.

MS (EI): m/z, (\%) = 368 (8), 367 (4), 366 (14), 339 (28), 337 (55), 335 (30), 230 (21), 228 (22), 150 (100), 149 (24), 129 (20), 115 (23), 83 (26), 81 (18), 75 (56), 74 (25), 71 (24).

\section{3,6-Dibromo-9,10-dimethoxyphenanthrene (33)}

A mixture of 3,6-dibromo-9,10-phenanthrenequinone 32 (2.5 g, $6.83 \mathrm{mmol})$, tetrabutylbutyl ammonium bromide (0.704 g, $2.186 \mathrm{mmol})$, sodium dithionite $(3.567 \mathrm{~g}, 20.49 \mathrm{mmol})$, THF (30 mL) and $\mathrm{H}_{2} \mathrm{O}(30 \mathrm{~mL})$ in a round bottom flask was stirred for $30 \mathrm{~min}$, then dimethyl sulfate $(3.36 \mathrm{~mL}$, $35.52 \mathrm{mmol}$ ) was added, followed by aqueous $\mathrm{NaOH}(3.56 \mathrm{~g}$ in $20 \mathrm{~mL}$ water). The mixture was stirred for $15 \mathrm{~min}$, while after $3 \mathrm{~min}, 5-6 \mathrm{~g}$ of ice was added to keep mixture at ambient temperature. The aqueous layer was separated and extracted with ethyl acetate, the combined organic layer was washed with water, dilute ammonia, brine and dried over sodium sulfate and concentrated under reduced pressure. The crude product was purified by column chromatography on silica gel using ethyl acetate: petroleum ether as eluent to obtain a colorless solid $(2.46 \mathrm{~g}, 86 \%)$, mp. $134-136{ }^{\circ} \mathrm{C} .{ }^{39 c}$ IR $v(\mathrm{KBr}) \mathrm{cm}^{-1}: 3438,2932,2837,2366,2340,1906,1720,1614,1587,1482,1458,1422,1394$, $1345,1311,1229,1202,1121,1091,1064,1023,985,861,820,707,601$.

${ }^{1} \mathrm{H}-\mathrm{NMR}\left(400 \mathrm{MHz}, \mathrm{CDCl}_{3}\right): \delta 8.56-8.57(\mathrm{~d}, \mathrm{~J}=1.44 \mathrm{~Hz}, 2 \mathrm{H}), 8.00-8.02(\mathrm{~d}, \mathrm{~J}=8.72 \mathrm{~Hz}, 2 \mathrm{H}), 7.63-$ $7.65(\mathrm{dd}, \mathrm{J}=8.72,1.44 \mathrm{~Hz}, 2 \mathrm{H}), 3.99(\mathrm{~S}, 6 \mathrm{H})$.

MS (EI): $m / z,(\%) 398$ (43), 396 (91), 394 (53), 382 (35), 380 (75), 378 (43), 352 (33), 337 (24), 273 (56), 271 (57), 165 (42), 163 (22), 150 (100), 148 (95), 81 (28).

9,10-Dimethoxy-3,6-distyrylphenanthrene (34) was obtained in $93 \%$ yield $(0.52 \mathrm{~g})$, on a 1.26 mmol scale, by (method A). It was purified by column chromatography on silica gel using petroleum ether as eluent to afford pale yellow solid, mp. $116-120^{\circ} \mathrm{C}$.

IR $v(\mathrm{KBr}) \mathrm{cm}^{-1}: 3438,3024,2931,2835,1602,1503,1446,1419,1364,1327,1243,1202,1121$, 1062, 986, 954, 816, 751 .

${ }^{1} \mathrm{H}-\mathrm{NMR}\left(400 \mathrm{MHz}, \mathrm{CDCl}_{3}\right): \delta 8.71-8.71(\mathrm{~d}, \mathrm{~J}=1.20 \mathrm{~Hz}, 2 \mathrm{H}), 8.21-8.23(\mathrm{~d}, \mathrm{~J}=8.40 \mathrm{~Hz}, 2 \mathrm{H}), 7.87-$ $7.89(\mathrm{dd}, \mathrm{J}=8.80,1.60 \mathrm{~Hz}, 2 \mathrm{H}), 7.62-7.64(\mathrm{~m}, 4 \mathrm{H}), 7.28-7.44(\mathrm{~m}, 10 \mathrm{H}$, overlapping the trans coupling proton signals $\mathrm{d}, \mathrm{J}=16.40 \mathrm{~Hz}), 4.11(\mathrm{~s}, 6 \mathrm{H})$.

MS (EI): m/z, (\%) 443 (33), 442 (100), 428 (13), 427 (41), 399 (23), 221 (53), 213 (10), 192 (10), 191 (14), 184 (15), 183 (13), 182 (18), 177 (23), 176 (34), 175 (12), 169 (32), 163 (11).

${ }^{13} \mathrm{C}-\mathrm{NMR}\left(50 \mathrm{MHz}, \mathrm{CDCl}_{3}\right): \delta 61.69\left(-\mathrm{OCH}_{3}, 2 \mathrm{C}\right), 122.25(-\mathrm{CH}, 2 \mathrm{C}), 123.29(-\mathrm{CH}, 2 \mathrm{C}), 125.17$ ($\mathrm{CH}, 2 \mathrm{C}), 127.25$ (-CH, 4C), 128.37 (-CH, 2C), 129.43 (-CH, 4C), 129.43 (-C, 4C) [DEPT 90 \& 135 analysis shows the decrease in the intensity of signal due to overlapping quaternary carbon.], 135.49 (-C, 2C), 138.04 (-C, 2C), 144.79 (-C, 2C).

9,10-Dimethoxy[7]helicene $\mathbf{( 3 5 )}$ by (method B). A solution of 3,6-distyryl-9,10dimethoxyphenanthrene $37(0.450 \mathrm{~g}, 1.02 \mathrm{mmol})$, iodine (0.568 g, $2.24 \mathrm{mmol}, 2.2$ eq.), tetrahydrofuran $(7.34 \mathrm{~g}, 8.25 \mathrm{~mL}, 10.18 \mathrm{mmol}, 100$ eq.) and toluene (1.2 L) was irradiated using a 250W HMPV lamp for $36 \mathrm{~h}$. After the reaction was over, the excess of iodine was removed by washing the solution with aqueous $\mathrm{Na}_{2} \mathrm{~S}_{2} \mathrm{O}_{3}$, followed by distilled water. The organic layer was 
concentrated under reduced pressure. After column chromatography, the pure product was obtained as orange-yellow crystals $(0.225 \mathrm{~g}, 50 \%)$, mp. $270-272{ }^{\circ} \mathrm{C}$, (Lit. $\left.>250{ }^{\circ} \mathrm{C}\right) .{ }^{40}$

IR $v(\mathrm{KBr}) \mathrm{cm}^{-1}:$ 3435, 3045, 2977, 2941, 2362, 1600, 1571, 1519, 1469, 1418, 1375, 1277, 1096, 1048, 987, 828, 749 .

${ }^{1} \mathrm{H}-\mathrm{NMR}\left(500 \mathrm{MHz}, \mathrm{CDCl}_{3}\right): \delta 8.37-8.39(\mathrm{~d}, \mathrm{~J}=8.00 \mathrm{~Hz}, 2 \mathrm{H}), 7.94-7.95(\mathrm{~d}, \mathrm{~J}=8.50 \mathrm{~Hz}, 2 \mathrm{H}), 7.71-$ $7.73(\mathrm{~d}, \mathrm{~J}=8.50 \mathrm{~Hz}, 2 \mathrm{H}), 7.46-7.48(\mathrm{~d}, \mathrm{~J}=8.00 \mathrm{~Hz}, 2 \mathrm{H}), 7.28-7.29(\mathrm{dd}, \mathrm{J}=8.50,1.00 \mathrm{~Hz}, 2 \mathrm{H})$, 7.12-7.14 (d, J = 8.00 Hz, 2H), 6.88-6.91 (ddd, J = 8.50, 8.00, 1.50 Hz, 2H), 6.37-6.41 (ddd, J = $8.50,7.00,1.50 \mathrm{~Hz}, 2 \mathrm{H}), 4.25(\mathrm{~s}, 6 \mathrm{H})$.

MS (EI): $m / z,(\%) 439$ (21), 438 (63), 423 (28), 395 (28), 380 (24), 350 (25), 203 (22), 195 (30), 181 (36), 180 (34), 175 (100), 174 (40), 168 (24), 111 (22), 97 (33), 85 (43), 84 (22), 83 (43), 71 (43).

Benzo $[c]$ phenanthrene (36) was obtained in $84 \%$ yield $(0.125 \mathrm{~g})$ on a $0.65 \mathrm{mmol}$ scale by (method B). It was purified by column chromatography on silica gel using petroleum ether as eluent to afford colorless solid, mp. $68-69^{\circ} \mathrm{C}$ (Lit. 67-68 $\left.{ }^{\circ} \mathrm{C}\right) .{ }^{41}$

IR $v(\mathrm{KBr}) \mathrm{cm}^{-1}:$ 3042, 3007, 2926, 1618, 1599, 1518, 1494, 1458, 1418, 832, 806, 744.

${ }^{1} \mathrm{H}-\mathrm{NMR}\left(200 \mathrm{MHz}, \mathrm{CDCl}_{3}\right): \delta 9.12-9.16(\mathrm{~d}, \mathrm{~J}=8.00 \mathrm{~Hz}, 2 \mathrm{H}), 8.01-8.05(\mathrm{dd}, \mathrm{J}=8.00,2.00 \mathrm{~Hz}$, 2H), 7.81-7.89 (dd, J = 8.00 Hz, 4H), 7.58-7.73 (m, 4H).

MS (EI): m/z, (\%) 230 (02), 229 (19), 228 (100), 227 (48), 226 (43), 113 (17), 112 (06).

2-Chlorobenzo[c]phenanthrene (37) was obtained in $67 \%$ yield $(0.10 \mathrm{~g})$ on a $0.567 \mathrm{mmol}$ scale by (method B). It was purified by column chromatography on silica gel using petroleum ether as eluent to afford colorless solid, mp. 62-64 ${ }^{\circ} \mathrm{C}$ (Lit. 61.4-61.8 ${ }^{\circ} \mathrm{C}$ ). ${ }^{41}$

IR $v(\mathrm{KBr}) \mathrm{cm}^{-1}:$ 3046, 2924, 1596, 1487, 1440, 1419, 1110, 1092, 1039, 838, 779, 747.

${ }^{1} \mathrm{H}-\mathrm{NMR}\left(200 \mathrm{MHz}, \mathrm{CDCl}_{3}\right): \delta 9.11(\mathrm{~d}, \mathrm{~J}=1.30 \mathrm{~Hz}, 1 \mathrm{H}), 9.03-9.06(\mathrm{~d}, \mathrm{~J}=9.70 \mathrm{~Hz}, 1 \mathrm{H}), 8.01-8.03$ $(\mathrm{dd}, \mathrm{J}=7.70,0.80 \mathrm{~Hz}, 1 \mathrm{H}), 7.93-7.95(\mathrm{~d}, \mathrm{~J}=8.50 \mathrm{~Hz}, 1 \mathrm{H}), 7.90-7.93(\mathrm{~d}, \mathrm{~J}=8.50 \mathrm{~Hz}, 1 \mathrm{H}), 7.80-$ $7.87(\mathrm{~m}, 3 \mathrm{H}), 7.71-7.75(\mathrm{~m}, 1 \mathrm{H}), 7.63-7.66(\mathrm{~m}, 1 \mathrm{H}), 7.56-7.58(\mathrm{dd}, \mathrm{J}=8.50,2.00 \mathrm{~Hz}, 1 \mathrm{H})$.

MS (EI): m/z, (\%) 265 (06), 264 (32), 263 (20), 262 (100), 228 (10), 227 (57), 226 (84), 225 (21), 224 (23), 113 (72), 112 (33).

\section{Acknowledgements}

We wish to thank Department of Science and Technology, New Delhi for the financial support (SR/SI/OC-42/2006; for HRT) and Council of Scientific and Industrial Research, New Delhi for the award of a fellowship (ARC). We are grateful to Prof. B.V. Kamath, Head, Department of Chemistry for the laboratory facilities. We also thank RSIC, Punjab University, Chandigarh and Apicore Pharmaceuticals Ltd. for recording the NMR spectra.

\section{References}

1. (a) Martin, R. H. Angew. Chem. Int. Ed. 1974, 13, 649. (b) Katz, T. J. Angew. Chem. Int. Ed. 2000, 39, 1921. (c) Schmuck, C. Angew. Chem. Int. Ed. 2003, 42, 2448. (d) Urbano, A. Angew. Chem. Int. Ed. 2003, 42, 3986. (e) Laarhoven, W. H.; Prinsen, W. J. C. Top. Curr. Chem. 1984, 
125, 63. (f) Vögtle, F. in Fascinating Molecules in Organic Chemistry; Wiley, New York, 1992; p 156. (g) Meurer, K. P.; Vögtle, F. Top. Curr. Chem. 1985, 127, 1. (h) Rowan, A. E.; Notle, R. J. M. Angew. Chem. Int. Ed. 1998, 37, 63. (i) Hopf, H. in Classics in Hydrocarbon Chemistry: Syntheses, Concepts, Perspectives; VCH: Weinheim, 2000; p 323. (j) Rajca, A.; Miyasaka, M. in Functional Organic Materials. Syntheses, Strategies, and Applications. WILEY-VCH Verlag GmbH \& Co. KGaA, Weinheim 2007, p 547.

2. (a) Verbist, T.; Van Elshocht, S.; Kauranen, M.; Hellemans, L.; Snauwaert, J.; Nuckolls, C.; Katz, T. J.; Persoons, A. Science, 1998, 282, 913. (b) Fox, J. M.; Katz, T. J.; Van Elshocht, S.; Verbiest, T.; Kauranen, M.; Persoons, M. A.; Thongpanchang, T.; Krauss, T.; Brus, L. J. Am. Chem. Soc. 1999, 121, 3453. (c) Van Elshochta, S.; Verbiest, T.; Busson, B.; Kauranen, M.; Snauwaert, J.; Hellemans, L.; Persoons, A.; Nuckolls, C.; Phillips, K. E.; Katz, T. J. Synthetic Metals 2000, 115, 201.

3. Phillips, K. E. S.; Katz, T. J.; Jockusch, S.; Lovenger, A.; Turro, N. J. J. Am. Chem. Soc. 2001, 123, 11899.

4. (a) Martin, R. H.; Marchant, M. J. Tetrahedron 1974, 30, 347. (b) Yamada, K.; Nakagawa, H.; Kawazura, H. Bull. Chem. Soc. Jpn., 1986, 59, 2429.

5. (a) Nuckolls; C.; Katz, T. J. J. Am. Chem. Soc. 1998, 120, 9541. (b) Hibert, M.; Solladie, G. J. Org. Chem. 1980, 45, 5393.

6. (a) Fox, J. M.; Lin, D J. Org. Chem. 1998, 63, 2031. (b) Dai, Y.; Katz, T. J. J. Org. Chem. 1997, 62, 1274. (c) Nuckolls, C.; Katz, T. J.; Verbiest, T.; Vanelshocht, S.; Kuball, H. G.; Kiesewalter, S.; Lovinger, A. J.; Persoons, A. J. Am. Chem. Soc. 1998, 120, 8656.

7. (a) Newman, M. S.; Darlak, R. S.; Tsai, L. L. J. Am. Chem. Soc., 1967, 89, 6191. (b) Pieters, G.; Gaucher, A.; Marque, S.; Maurel, F.; Lesot, P.; Prim, D. J. Org. Chem. 2010, 75, 2096. (c) Morrison, D. J.; Trefz, T. K.; Piers, W. E.; McDonald, R.; Parvez, M. J. Org. Chem. 2005, 70, 5309.

8. For Alcohols and its derivatives: (a) Yamamato, K.; Ikeda, T.; Kitsuki, T.; Okamoto, Y.; Chikamatsu, H.; Nakazaki, M. J. Chem. Soc. Perkin Trans. 1990, 1, 271. (b) Tanaka, K.; Osuga, H.; Shogase, Y.; Suzuki, H. Tetrahedron Lett. 1995, 36, 915. (c) Dreher, S. D.; Katz, T. J.; Lam, K. C.; Rheingold, A. L. J. Org. Chem. 2000, 65, 815. (d) Ben Hassine, B.; Gorsane, M.; Pecher, J.; Martin, R. H. Bull. Soc. Chim. Belg. 1985, 94, 597. (e) Ben Hassine, B.; Gorsane, M.; Pecher, J.; Martin, R.H. Bull. Soc. Chim. Belg. 1987, 96, 801. (f) Reetz, M. T.; Sostmann, S. Tetrahedron 2001, 57, 2515. (g) Carrño, M. C.; Garcia-Cerrada. S.; Urbano. A. J Am. Chem. Soc, 2001, 123, 7929. (h) Ogawa, Y.; Toyama, M.; Kirikomi, M.; Seki, K.; Haga, K.; Uyehara, T. Tetrahedron Lett. 2003, 44, 2167. (i) Teply, F.; Stará, I. G.; Starý, I.; Kollarovic, A.; Lustinec, D.; Krausova, Z.; Saman, D.; Fielder, P. Eur. J. org. Chem. 2007, 4244. (j) Aloui, F.; Abed, R. E.; Marinetti, A.; Ben Hassine, B. C. R. Chim. 2009, 12, 284. (k) Osuga, H.; Suzuki, H.; Tanaka, K. Bull. Chem. Soc. Jpn. 1997, 70, 891. (1) Aloui, F.; Abed, R. E.; Marinetti, A.; Ben Hassine, B. Tetrahedron Lett. 2008, 49, 4092. (m) Gingras, M.; Collet, C. Synlett, 2005, 2337.

9. For Nitriles and its derivatives: (a) Ben Hassine, B.; Gorsane, M.; Pecher, J.; Martin, R. H. Bull. Soc. Chim. Belg. 1986, 95, 547. (b) Stammel, C.; Frohlich, R.; Wolff, C.; Wenck. H.; de. 
Meijere, A.; Matty, J. Eur. J. Org. Chem. 1999, 1709. (c) Okubo, H.; Nakano, D.; Yamaguchi, M.; Kabuto, C. Chem. Lett. 2000, 1316.

10. For Amines and its derivatives: (a) Takenaka, N.; Chen, J.; Captain, B.; Singh Sarangthem, R.; Chandrakumar, A. J. Am. Chem. Soc. 2010, 132, 4536. (b) Teply, F.; Stará, I. G.; Starý, I.; Kollarovic, A.; Saman, D.; Askocil, S. V.; Fiedler, P. J. Org. Chem. 2003, 68, 5193. (c) Perzyna, A.; Dal Zotto, C.; Durand, J. -O.; Granier, M.; Smietana, M.; Melnyk, O.; Stará, I. G.; Starý, I.; Klepetářová, B.; Aman, D. S. Eur. J. Org. Chem. 2007, 4032. (d) den Brouw, M, Op.; Larrhoven, W. H. Recl. Trav. Chim. Pays-Bas. 1978, 97, 265.

11. For Heliphos: (a) Reetz, M. T.; Beuttenmuller, E. W.; Goddard, R. Tetrahedron Lett. 1997, 38, 3211. (b) Terfort, A.; Gorls, H.; Brunner, H. Synthesis 1997, 79. (c) Reetz, M. T.; Sostmann, S. J. Organomet. Chem. 2000, 603, 105. (d) Paruch, K.; Vyklicky', L.; Wang, D.Z.; Katz, T. J.; Incarvito, C.; Zakharov, L.; Rheingold, A. L. J. Org. Chem. 2003, 86, 8539. (e) Aloui, F.; Abed, R. E.; Marinetti, A.; Ben Hassine, B. Tetrahedron Lett. 2007, 48, 2017. (f) Aloui, F.; Abed, R. E.; Marinetti, A.; Ben Hassine, B. Tetrahedron Lett. 2007, 48, 1156. (g) Nakano, D.; Yamaguchi, M. Tetrahedron Lett., 2003, 44, 4969. (i) Amemiya, R.; Yamaguchi, M. Org. Biomol. Chem., 2008, 6, 26. (h) Aloui, F.; Ben Hassinea, B. Tetrahedron Lett. 2009, 50, 4321.

12. Kawasaki, T.; Suzuki, K.; Licandro, E.; Bossi, A.; Maioranab, S.; Soai, K. Tetrahedron Asymmetry 2006, 17, 2050.

13. Kamikawa, K.; Takemoto, I.; Takemoto, S.; Matsuzaka, H. J. Org. Chem. 2007, 72, 7406.

14. (a) Kim, C.; Marks, T. J.; Facchetti, A.; Schiavo, M.; Bossi, A.; Maiorana, S.; Licandro, E.; Todescato, F.; Toffanin, S.; Muccini, M.; Graiff, C.; Tiripicchio, A. Organic Electronics 2009, 10, 1511. (b) Wang, Z. Y.; Todd, E. K.; Meng, X. S.; Gao, J. P. J. Am. Chem. Soc. 2005, 127, 11552. (c) Grimme, S.; Harrenb, J.; Sobanski, A.; Vögtle, F. Eur. J. Org. Chem. 1998, 1491. (d) Norel, L.; Rudolph, M.; Vanthuyne, N.; Williams, J. A. G.; Lescop, C.; C. Roussel, C.; Autschbach, J.; Crassous, J.; Rau, R. Angew. Chem. Int. Ed. 2010, 49, 99. (e) Bossia, A.; Falciola, L.; Graiff , C.; Maioranaa, S.; Rigamontia, C.; Tiripicchioc, A.; Licandroa, E.; Mussini, P. R. Electrochimica Acta 2009, 54, 5083.

15. (a) Flammang-Barbieux, M.; Nasielski, J.; Martin, R. H. Tetrahedron Lett. 1967, 8, 743. (b) Liu, L.; Yang, B.; Katz, T. J.; Poindexter, M. K. J. Org. Chem. 1991, 56, 3769. (c) Pearson, M. S. M.; Carbery, D. R. J. Org. Chem. 2009, 74, 5320.

16. (a) Abbate, S.; Bazzini, C.; Caronna, T.; Fontana, F.; Gambarotti, C.; Gangemi, F.; Longhi, G.; Mele, A.; Sora, I. N.; Panzeri, W. Tetrahedron 2006, 62, 139. (b) Bazzini, C.; Brovelli, S.; Caronna, T.; Gambarotti, C.; Giannone, M.; Macchi, P.; Meinardi, F.; Mele, A.; Panzeri, W.; Recupero, F.; Sironi, A.; Tubino, R. Eur. J. Org. Chem. 2005, 1247.

17. Talele, H. R.; Gohil, M. J.; Bedekar, A. V. Bull. Chem. Soc. Jpn. 2009, 82, 1182.

18. (a) Fried, S.; Kleene, R. D. J. Am. Chem. Soc. 1941, 63, 2691. (b) Vogel's textbook of quantitative chemical analysis, 5th Ed. 1989, Longman Scientific \& Technical, UK.

19. (a) Wood, C. S.; Mallory, F. B. J. Org. Chem 1964, 29, 3373. (b) Laarhoven, W. H. Recl. Trav. Chim. Pays-Bas. 1983, 102, 185. 
20. Sorenson, R. J.; Buranzu R. E.; Anangusuto, P. S.; Shiyuuendaa, C. E.; Keazaazu, M. I.; Shitenko, U. E.; Konaa D. T.; Jiyonson, E. E.; Kiirii, J. E.; Saakaa J. S. JP62081369, 1987 (CAN 107, 197805).

21. (a) Dietz, F.; Scholz, M. Tetrahedron 1968, 24, 6845. (b) Laarhoven, W. H.; Cuppen, T. J. H. M.; Nivard, R. J. F. Tetrahedron 1970, 26, 1069. (c) Xue, X.; Scott, L. T. Org. Lett. 2007, 9, 3937, also see $9 b$.

22. (a) Carbi, W.; Candiani, I.; Bedeschi, A.; Penco, S.; Santi, R. J. Org. Chem. 1992, 57, 1481. (b) Carbi, W.; Candiani, I.; Bedeschi, A. J. Org. Chem. 1992, 57, 3558.

23. Martin, R. H.; Flammang-Barbieux, M.; Cosyn, J. P.; Gelbcke, M. Tetrahedron Lett., 1968, 31, 3507.

24. Chaudhary, A. R.; Bedekar, A. V. Synth. Commun. 2011, 42, 0000.

25. (a) Abed, R. E.; Aloui, F.; Genet, J. -P.; Ben Hassine, B.; Marinetti, A. Journal of Organometallic Chemistry 2007, 692, 1156. (b) Amsharov, K. Y.; Kabdulov, M. A.; Jansen, M. Eur. J. Org. Chem. 2009, 6328. (c) Aloui, F.; Abed, R. E.; Ben Hassine, B. Tetrahedron Lett. 2008, 49, 1455. (d) Blackburn, E. V.; Loader, C. E.; Timmons, C. J. J. Chem. Soc. C, 1968, 1576.

26. (a) Filimonov, V. D.; Trusova, M.; Postnikov, P.; Krasnokutskaya, E. A.; Lee, Y. M.; Hwang, H. Y.; Kim, H.; Chi, K. W. Org. Lett. 2008, 10, 3961. (b) Hunter, D. H.; Cram, D. J. J. Am. Chem. Soc. 1966, 88, 5765. (c) Huang, S. -H.; Chen, J. -R.; Tsai, F. -Y. Molecules 2010, 15, 315.

27. (a) Judd, M. C.; Hartshorn, M. P.; Martyn, R. J.; Robinson, W.; Wright, G. J.; Vannoort, R. W. Aust. J. Chem. 1990, 43, 125. (b) Gammans, R. E.; Sehon, R. D.; Anders, M. W.; Hanna, P. E. Drug. Metab. Dispos. 1977, 5, 310.

28. Lapouyade, R.; Veyers, A.; Hanafi, N.; Couture, A.; Lablache-Combier, A. J. Org. Chem. 1982, 47, 1361.

29. Scholz, M.; Muhlstadt, M.; Dietz, P. Tetrahedron lett. 1967, 30, 665.

30. Brooks, M. A.; Scott, L. T. J. Am. Chem. Soc. 1999, 121, 5444.

31. Martin, R. H.; Moriau, J.; Defay, N. Tetrahedron 1974, 30, 179.

32. Hibert, M.; Solladie, G. J. Org. Chem. 1980, 45, 5393.

33. Laarhoven, W. H.; Cuppen, T. J. H. M.; Nivard, R. J. F. Tetrahedron 1970, 26, 4865.

34. Laarhoven, W. H.; Veldhuis, R. G. M. Tetrahedron, 1972, 28, 1811.

35. Porzi, G.; Concilio, C. J. Organomet. Chem., 1977, 128, 95.

36. Martin, R. H.; Marchant, M. -J.; Baes, M. Helv. Chim. Acta, 1971, 54, 358.

37. Lightner, D. A.; Hefelfinger, D. T.; Powers, T. W. P.; Frank, G. W.; Trueblood, K. N. J. Am. Chem. Soc. 1972, 94, 3492.

38. Abed, R. E.; Ben Hassine, B.; Genêt, J. -P.; Gorsane, M.; Marinetti, A. Eur. J. Org. Chem. 2004, 1517.

39. (a) Wendland, R.; LaLonde, J. Organic Syntheses, Coll. Vol. 4, 1963, p.757. (b) Bhatt, M. V. Tetrahedron, 1964, 20, 803. (c) Boden, B. N.; Jardine, K. J.; Leung, A. C. W.; MacLachlan, M. J. Org. Lett. 2006, 8, 1855.

40. Harrowven, D. C.; Guy, I. L.; Nanson, L. Angew. Chem. Int. Ed. 2006, 45, 2242. 
41. Amsharov, K.Y.; Kabdulov, M.A.; Jansen, M. Eur. J. Org. Chem. 2009, 36, 6328. 